\title{
EFFECT OF TURBULENCE AND CHANNEL SLOPE ON TRANSLATION WAVES
}

\author{
By Garbis H. Keulegan and George W. Patterson
}

\section{ABSTRACT}

This paper is the second of a series dealing with the motion of flood waves and other waves of translation in open channels. The first paper considered waves controlled solely by inertia forces; the present one is an analysis of the combined effects of turbulent friction and inertia. The basic equation of motion for gradually varied unsteady flow in prismatic channels is derived from fundamental principles. The effect of the velocity distribution in the original undisturbed current on the motion of short waves is investigated, and the effects of wave height, curvature of profile, and fluid friction on the celerity of a wave-volume element is analyzed in detail. The deformation of a straight sloping front and the change of height of an abrupt wave front is treated. Special emphasis is laid on disturbances of negligible curvature and practical methods of handling engineering problems arising in connection with the operation of locks or hydroelectric canals are given.

\section{CONTENTS}

List of symbols ...

I. Introduction

II. Fundamental principles_.

1. Reynolds equation of mean motion

2. Equation of continuity for mean flow in channels . . .

III. First-order theory of wave propagation

1. The equation of mean flow in prismatic channels

2. Coefficient of resistance as a function of $H_{\ldots} \ldots \ldots \ldots$

3. Velocity of propagation of short waves of negligible curvature _ 478

4. Propagation of discontinuities of slope

5. Effect of velocity distribution on the celerity of short waves ... 486

IV. Second-order theory of wave propagation ........ 490

1. Equation of mean flow with appreciable vertical acceleration _.- 490

2 . Height of wave as a function of $x$ and $t \ldots \ldots \ldots$.............. 491

3 . Celerity of a wave-volume element.................... 493

4. Deformation of the wave profile, assuming $g H i-\lambda U^{2} / 2=0 \ldots 495$

5 . Effect of friction and slope of bed on the propagation of waves_. 496

6. Deformation of a straight sloping front

7. Change of height of an abrupt wave front

8. Solution of the wave equation when slope and friction are not negligible _....... 503

9. Intumescence produced by operating locks in a canal carrying water

10. Effect of friction on waves when the slope of the bed vanishes_- 507

11. Solution of the wave equation when the slope vanishes but friction is considered

12. Intumescence produced by operating locks in an ordinary canal 510

13. Limitations of Boussinesq solutions _. .

V. References................ 512 


\section{LIST OF SYMBOLS}

Symbols used in only one section are omitted unless they are of general significance. Important quantities in hydraulic equations are indicated as functions of $x$ and $t$. All equations are valid in any consistent set of units. The significance of barred variables is explained in section II-1.

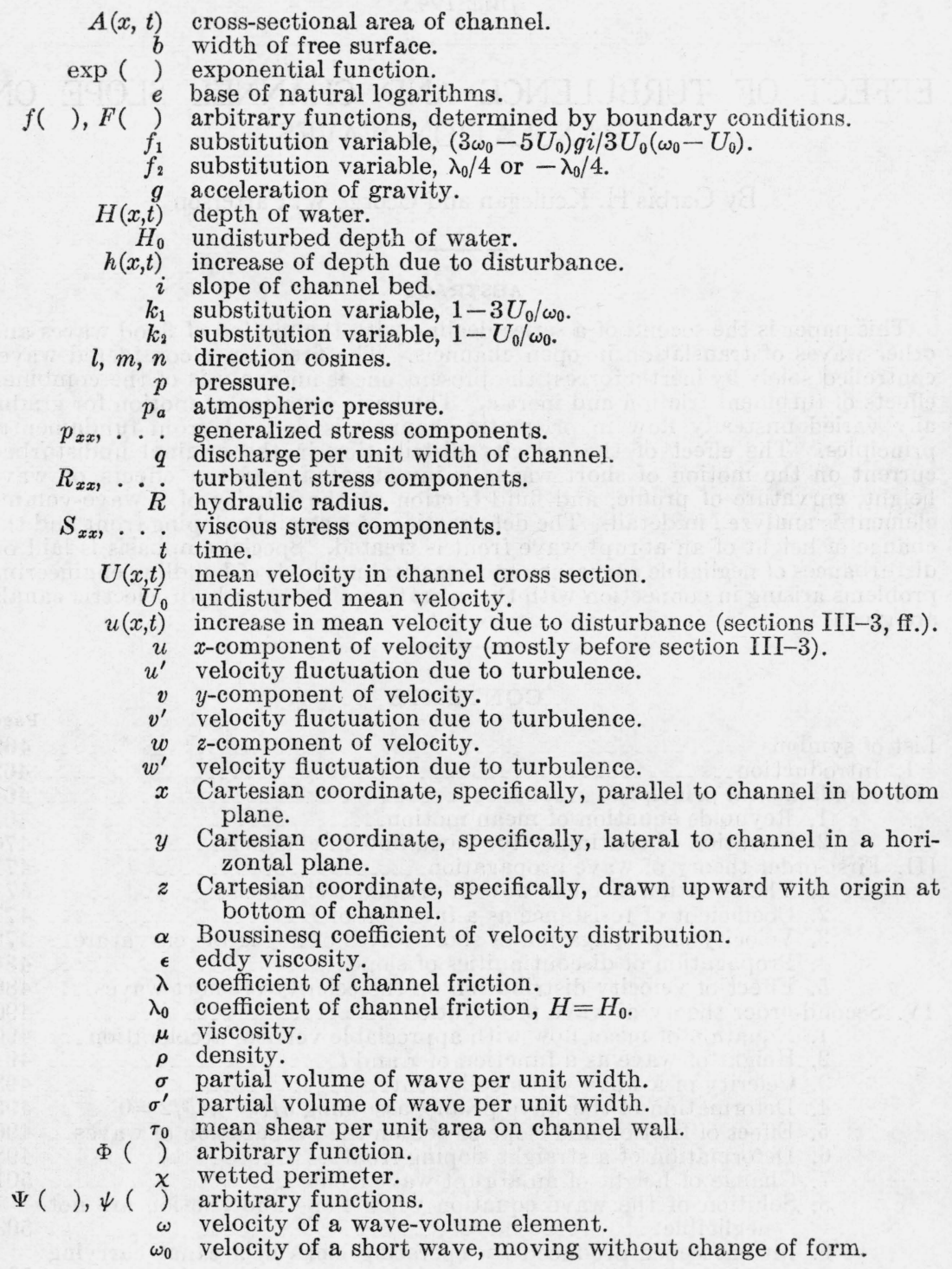

\section{INTRODUCTION}

This paper is the second of a series dealing with the motion of flood waves and other waves of translation in open channels. The purpose of the series is explained in the introduction to the first paper [1]. ${ }^{1}$

1 Figures in brackets indicate the literature references at the end of this paper. 
Many of the significant properties of translation waves may be ob tained by assuming that the motion of fluid particles due to the wave disturbance is irrotational [1]. Experimental observation of these properties indicates that they are in good agreement with theoretical predictions, provided that the waves are moving in still water. If, on the other hand, the disturbances are produced in a liquid which is initially in motion, as in actual currents in an open channel, some disparity will be seen between observation and theory. Bazin's ex periments on the velocity of propagation of a solitary wave in still water or in a running current demonstrate these points [2].

The divergence between observation and theory, when the wave is moving in a current, can be explained in two ways. First, irrotational or potential theory supposes that the original undisturbed motion is such that all the particles in a cross section move with the same constant velocity, $U_{0}$, which may of course be zero. This condition is not fulfilled for flow in actual channels, and it thus becomes necessary to investigate the effect of the distribution of velocity in a cross section on the velocity of propagation of waves. An analysis of this effect has been carried through in great detail by Boussinesq [3]. With a view to simplifying the presentation and the solution of the problem, the authors give a new derivation (section III-5). Although the results arrived at here are different in form from those of Boussinesq, it is believed that the difference has a qualitative rather than quantitative importance.

This difference is due to two distinct forms of the equation of motion of unsteady flow in a channel, one adopted by Boussinesq and the other by the authors. The present method applies the theory of Reynolds stresses to the problem of the unsteady mean flow in a channel, without assuming any specific relation between the stresses and the velocity rates existing at points within the channel cross section. The effect of these stresses is evaluated in terms of the total friction at the wall, which is the important concept for mean flow in open channels and is usually available from observations on uniform flow. Boussinesq's method on the other hand is an extension of his own theory of turbulent flow, and specific relations between the turbulent stresses and the space-rates of the local velocities are assumed and introduced into the derivations. As the concept of Reynolds stresses is the starting point of the present analysis, the theory of these stresses is given in considerable detail (section II-1). The equation of motion of a perfect fluid is the point of departure in interpreting the relation between the stresses and velocity fluctuations. This novel procedure simplifies the presentation considerably. It is hoped that the equation of motion for gradually varied unsteady flow in prismatic channels derived in this paper, eq 47 , is sufficiently rigorous to merit the attention of river engineers.

In the second place, the irrotational theory of translation waves ignores the effect of turbulence, which is one of the forces controlling the motion of the waves. Consider a short intumescence moving in a current. In the initial stages of motion the behavior of the wave is not affected by turbulence, since the wave is assumed to be short. When the wave has traveled a considerable distance the cumulative effect of friction becomes appreciable, particularly at the rear of the wave. In fact, experience shows that the front of the wave will travel with a velocity characteristic of irrotational waves, whereas at 


\section{Journal of Research of the National Bureau of Standards}

the other end of the wave, the volume elements will move with a smaller velocity, the exact law of which is not as yet clearly understood. The wave thus flattens out considerably at the posterior end, with a consequent elongation. The tendency of short waves to deform in this manner is clearly demonstrated in Horton's experiments [4].

When the motion of the waves is controlled principally by friction, as in the quasipermanent regimes of rivers, the corresponding theory of translation waves is simple. But when it is supposed that the friction and inertia effects are of like magnitude, analysis is confronted with a difficult task. The change of shape of a short intumescence during the entire journey of the wave in a current would be accurately described if a complete solution were available, which is not the case. However, some progress in this direction is possible if it is assumed that the effect of turbulence is small, requiring only a secondary correction. Such a theory has been worked out by Boussinesq, who assumed that the velocity of the wave-volume elements differs little from the theoretical velocity of waves without friction [3]. The main purpose of this paper is to consider this theory and the corresponding solutions for waves of negligible curvature. In giving Boussinesq's solution, slight changes have been made, since it was desired to adopt Manning's law of friction in open channels [5]. Favre has employed the Boussinesq integrals to describe the motion of waves occurring during the operation of locks [6]. Because of the engineering significance of the problem we have also reproduced these applications (section IV-9 and IV-12).

The applications of the theory are presented in sections III and IV, which have been entitled first-order and second-order theories of wave propagation. The first-order theory neglects the vertical acceleration of the water particles and assumes that the combined effect of friction and velocity is small. It is found that this assumption requires that the waves be short. The second-order theory considers the effects of vertical acceleration but neglects the variations of the horizontal velocity components. As in Boussinesq's investigations, the concept of the velocity of a wave-volume element plays a very basic role. Equation 116 is the general expression for this velocity. Using this same formula, the authors have been able to study the gradual deformation of a straight sloping front and also to compute the reduction of height of an abrupt wave front.

\section{FUNDAMENTAL PRINCIPLES}

\section{REYNOLDS EQUATIONS OF MEAN MOTION}

In the Reynolds theory of turbulence $[7 ; 8$, p. $638 ; 9$, p. $364 ; 10$, p. 191] the velocity components $u, v, w$, in the directions $x, y, z$, respectively, are regarded as fluctuating functions of the variables $x, y, z, t$. The fluctuations are frequent and rapid, and the periodicity of the changes is irregular. Denoting the mean values of $u, v, w$ by $\bar{u}, \bar{v}, \bar{w}$, and the mean value of the pressure, $p$, by $\bar{p}$, we have for the instantaneous values

and

$$
\left.\begin{array}{c}
u=\bar{u}+u^{\prime}, \\
v=\bar{v}+v^{\prime}, \\
w=\bar{w}+w^{\prime}, \\
p=\bar{p}+p^{\prime},
\end{array}\right\}
$$


where $u^{\prime}, v^{\prime}, w^{\prime}$, and $p^{\prime}$ are the fluctuations whose mean values $\overline{u^{\prime}}, \overline{v^{\prime}}$, $\overline{w^{\prime}}, \overline{p^{\prime}}$ are equal to zero. The mean values may be temporal; that is, taken at a given point $(x, y, z)$ over an interval of time of duration $\Delta t$; thus, for example,

$$
\bar{u}=\frac{1}{\Delta t} \int_{t}^{t+\Delta t} u d t .
$$

Or again the mean values may be spatial; that is, taken at a given instant $t$ over a space of volume $\Delta V$; thus, for example

$$
\bar{u}=\frac{1}{\Delta V} \int \Delta v u d V \text {. }
$$

For the present it will be supposed that the averages are temporal. A difficulty arises when taking temporal means in unsteady flow. In such cases it is necessary to make the time interval $\Delta t$ long enough to include a great number of fluctuations, but short enough to exclude all but negligible variations of $\bar{u}, \bar{v}, \bar{w}, \bar{p}$.

There are certain rules for taking the averages. Expressed symbolically $[9$, p. 366]

and

$$
\left.\begin{array}{c}
\overline{a b}=\bar{a} \bar{b}, \\
\overline{a+b}=\bar{a}+\bar{b}, \\
\overline{\bar{a}}=\bar{a} \\
\frac{\partial a}{\partial s}=\frac{\partial}{\partial s} \overline{(a)}
\end{array}\right\}
$$

where $a$ and $b$ are functions of $x, y, z, t ; s$ represents any one of the independent variables, and a bar over a quantity represents an average of the kind just described. These rules are based upon assumptions that are not exactly applicable to the flow of liquids. The errors, however, are negligible when the fluctuations within the time interval $\Delta t$ are sufficiently numerous.

The specific contribution of Reynolds' analysis is the idea of interpreting the dynamic effects of the fluctuations $u^{\prime}, v^{\prime}, w^{\prime}$, as apparent tractional forces or stresses. The nature and the magnitude of these stresses can be readily obtained starting with the equations of motions of a perfect fluid. The dynamic equations in question are

$$
\left.\begin{array}{l}
\rho \frac{d u}{d t}=-\frac{\partial p}{\partial x}+\rho X, \\
\rho \frac{d v}{d t}=-\frac{\partial p}{\partial y}+\rho Y, \\
\rho \frac{d w}{d t}=-\frac{\partial p}{\partial z}+\rho Z,
\end{array}\right\}
$$

where the operator $d / d t$, in terms of the local variations, is

$$
\frac{d}{d t}=\frac{\partial}{\partial t}+u \frac{\partial}{\partial x}+v \frac{\partial}{\partial y}+w \frac{\partial}{\partial z} \text {. }
$$


Together with these, there is the equation of continuity, assuming that the liquid is incompressible,

$$
\frac{\partial u}{\partial x}+\frac{\partial v}{\partial y}+\frac{\partial w}{\partial z}=0
$$

The liquid will always be assumed to be incompressible, in this paper. In eq $5, p$ is the pressure, $\rho$ the density of liquid, and $X, Y, Z$ the components of the force of gravity per unit mass of liquid in the direction of $x, y, z$. These relations are valid at any instant of time independent of the fact that the quantities, $u, v, w$, and $p$ are fluctuating functions of time, provided that the velocities are not discontinuous, and if discontinuities exist, they occur only occasionally during the time interval $\Delta t$.

Replacing $u, v, w$ in eq 7 by their respective values from eq 1 , averaging each term, and using the last three relations in eq 4, together with the fact that $\bar{u}^{\prime}=\bar{v}^{\prime}=\bar{w}^{\prime}=0$, we obtain

$$
\frac{\partial u}{\partial x}+\frac{\partial v}{\partial y}+\frac{\partial w}{\partial z}=0
$$

which states that the dilatation of the mean values of the velocity components vanish. It then follows from eq 1, 7, 8 that

$$
\frac{\partial u^{\prime}}{\partial x}+\frac{\partial v^{\prime}}{\partial y}+\frac{\partial w^{\prime}}{\partial z}=0
$$

The equations of motion, eq 5 , are treated in a similar manner. We use 6 , substitute for $u, v, w$ their values from eq 1 , multiply eq 9 by $\rho u^{\prime}, \rho v^{\prime}, \rho w^{\prime}$, respectively, and add to the left-hand side of each equation. Each term is then averaged in accordance with eq 4 , assuming $\rho$ to be constant, that is, that the fluid is homogeneous, obtaining

$$
\left.\begin{array}{r}
\rho \overline{d \bar{u}}=-\frac{\partial \bar{p}}{\partial x}+\rho X+\frac{\partial R_{x x}}{\partial x}+\frac{\partial R_{y x}}{\partial y}+\frac{\partial R_{z x}}{\partial z} \\
\rho \overline{d \bar{v}}=-\frac{\partial \bar{p}}{\partial y}+\rho Y+\frac{\partial R_{x y}}{\partial x}+\frac{\partial R_{y y}}{\partial y}+\frac{\partial R_{z y}}{\partial z}, \\
\rho \frac{d \bar{w}}{d t}=-\frac{\partial \bar{p}}{\partial z}+\rho Z+\frac{\partial R_{x z}}{\partial x}+\frac{\partial R_{y z}}{\partial y}+\frac{\partial R_{z z}}{\partial z},
\end{array}\right\}
$$

where, in this case,

and

$$
\frac{d}{d t}=\frac{\partial}{\partial t}+\bar{u} \frac{\partial}{\partial x}+\bar{v} \frac{\partial}{\partial y}+\bar{w} \frac{\partial}{\partial z}
$$

$$
\left.\begin{array}{l}
R_{x x}=-\rho \overline{u^{\prime} u^{\prime}} ; R_{x y}=R_{y x}=-\rho \overline{u^{\prime} v^{\prime}} \\
R_{y y}=-\rho \overline{v^{\prime} v^{\prime}} ; R_{x z}=R_{z x}=-\rho \overline{u^{\prime} w^{\prime}} \\
R_{z z}=-\rho \overline{w^{\prime} w^{\prime}} ; R_{y z}=R_{z y}=-\rho \overline{v^{\prime} w^{\prime}} .
\end{array}\right\}
$$

We may refer to eq 10 as the Reynolds equations of motion for turbulent flow. 
To interpret the above results we write the general equations of motion of a deformable continuum under the action of the external body forces $X, Y, Z$ and surface tractions of an unspecified physical origin:

$$
\left.\begin{array}{l}
\rho \frac{d u}{d t}=\rho X+\frac{\partial p_{x x}}{\partial x}+\frac{\partial p_{y x}}{\partial y}+\frac{\partial p_{z x}}{\partial z} \\
\rho \frac{d v}{d t}=\rho Y+\frac{\partial p_{x y}}{\partial x}+\frac{\partial p_{y y}}{\partial y}+\frac{\partial p_{z y}}{\partial z}, \\
\rho \frac{d w}{d t}=\rho Z+\frac{\partial p_{x z}}{\partial x}+\frac{\partial p_{y z}}{\partial y}+\frac{\partial p_{z z}}{\partial z},
\end{array}\right\}
$$

where the operator $d / d t$ is the one defined in eq 6 .

It should be noted that these equations are merely Newton's law of motion as applied to any deformable continuum, and thus they apply to a wide range of phenomena. The left-hand members represent components of the rate of change of momentum per unit volume of the medium, whereas the right-hand members represent the components of the forces acting per unit volume. The nature of the medium determines the functional relation between the nine stress components, $p_{x x}, p_{y x}, \ldots \ldots$, and the other variables occurring in the equations and the intrinsic properties of the medium. Hydraulic equations established by integrating these equations over a cross section of the fluid normal to the flow are frequently referred to as momentum equations.

The nine stress components completely determine the forces acting on a small surface $d S$ at the point $(x, y, z)$. If a normal is drawn to $d S$ away from the body of which $d S$ forms a part of the surface, and if $l$, $m, n$ are the direction cosines of the normal to $d S$, then the $x-, y-, z-$ components of the surface tractions on the body at the point $(x, y, z)$ are

and

$$
\left.\begin{array}{l}
F_{x}=l p_{x x}+m p_{y x}+n p_{z x}, \\
F_{y}=l p_{x y}+m p_{y y}+n p_{z y}, \\
F_{z}=l p_{x z}+m p_{y z}+n p_{z z} .
\end{array}\right\}
$$

Comparing eq 10 and 13, we see that

$$
\left.\begin{array}{ll}
p_{x x}=-\bar{p}+R_{x x}, & p_{x y}=p_{y x}=R_{x y}=R_{y x} ; \\
p_{y y}=-\bar{p}+R_{y y}, & p_{x z}=p_{z x}=R_{x z}=R_{z x} ; \\
p_{z z}=-\bar{p}+R_{z z}, & p_{z y}=p_{y z}=R_{z y}=R_{y z} ;
\end{array}\right\}
$$

and thus the turbulent mean flow may be treated as ordinary streamline flow where the stresses $p_{x x}, p_{x y} \ldots \ldots$, are identified with the stresses $R_{x x}, R_{x y} \ldots$. These apparent stresses of turbulence are called Reynolds stresses, and represent mathematically the transport of momentum across a surface caused by the velocity fluctuations [10, p. 192].

If the flow instead of being turbulent is viscous in character, the stresses are of molecular origin. The stresses $p_{x x}, p_{x y}, \ldots$ are then 
identified with the viscous stresses $S_{x x}, S_{x y}, \ldots$ in the following manner,

$$
\left.\begin{array}{ll}
p_{x x}=-p+S_{x x}, & p_{x y}=p_{y x}=S_{x y}=S_{y x} ; \\
p_{y y}=-p+S_{y y}, & p_{x z}=p_{z x}=S_{x z}=S_{z x} ; \\
p_{z z}=-p+S_{z z}, & p_{y z}=p_{z y}=S_{y z}=S_{z y} .
\end{array}\right\}
$$

If the liquid is incompressible, these viscous stresses become

$$
\left.\begin{array}{l}
S_{x x}=2 \mu \frac{\partial u}{\partial x} ; \quad S_{x y}=S_{y x}=\mu\left(\frac{\partial v}{\partial x}+\frac{\partial u}{\partial y}\right) ; \\
S_{\nu y}=2 \mu \frac{\partial v}{\partial y}, \quad S_{z x}=S_{x z}=\mu\left(\frac{\partial w}{\partial x}+\frac{\partial u}{\partial z}\right) ; \\
S_{z z}=2 \mu \frac{\partial w}{\partial z}, \quad S_{y z}=S_{z \nu}=\mu\left(\frac{\partial v}{\partial z}+\frac{\partial w}{\partial y}\right) ;
\end{array}\right\}
$$

where $\mu$ is the viscosity of the liquid.

It is perfectly feasible to carry through the derivation of the Reynolds equations of motion, eq 10, using the viscous-flow interpretation of eq 13 as a starting point, and finally neglecting the viscous stresses in comparison with the Reynolds stresses, which is justifiable on the basis of experimental evidence [11, p. 186]. We have not adopted this method, since the results are identical with those obtained here by initially assuming a perfect liquid, that is, by neglecting the viscous stresses at the beginning rather than at the end, and since it is more complicated than the method used here.

Later, in sections III-1 and IV-1, we shall derive hydraulic equations for mean flow in open channels, using eq 10 as a starting point. For this purpose, it is sufficient merely to suppose that $R_{x x}, R_{x y} \ldots$. exist, there being no necessity for determining them as functions of the mean velocity gradients, because when the Reynolds equations of motion are integrated over the cross section of the channel, the quantities $R_{x x}, R_{x y}, \ldots$ either disappear or are expressed in terms of the wall friction, $\tau_{0}$, which is defined in connection with eq 45 . Boussinesq, however, expressed $R_{x x}, R_{x y}, \ldots$ in terms of the velocity gradients. He simulated eq17, merely replacing $S_{x x}, S_{x y}, \ldots$ by $R_{x x}, R_{x y}, \ldots ; u, v, w$ by $\bar{u}, \bar{v}, \bar{w}$; and $\mu$ by $\epsilon$. The quantity $\epsilon$ was called the coefficient of internal friction by Boussinesq [3, p. 46]; present usage seems to favor the term eddy viscosity, since $\epsilon$ has the same dimensions as $\mu[11$, p. 185$]$.

When the channel is rectangular, Boussinesq assumed that $\epsilon$ is constant for every point in a given cross section, but in a channel of constant roughness, directly proportional to the product of the wall velocity and the depth of the liquid. Such assumptions form the foundation for the developments in his "Essay." The validity of his theory is thus open to question, since the assumptions concerning $\epsilon$ are widely at variance with present experimental knowledge.

Having established the basic partial differential equations governing turbulent flow in open channels, we now turn our attention to the boundary conditions. Consider first the condition to be satisfied at a fixed solid wall, which we shall suppose is covered with uniform asperities of height $k$. A pillbox is erected on the surface, with height $h$, just sufficient to clear the asperities, and radius $r$, large with respect to $2 h$. (See fig. 1.) 


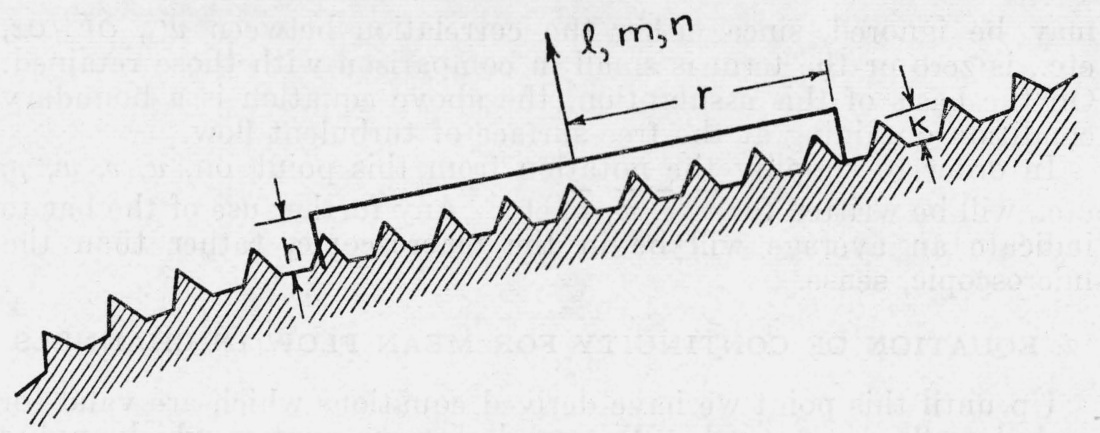

FiguRe 1.-A pillbox enclosing the asperities on a channel wall.

Since the liquid is incompressible, the net rate of flow across the surface of the pillbox must be zero. We neglect the discharge from the cylindrical side of the pillbox, since its area is small compared with that of the circular end. There must be at least one point on the end of the pillbox for which

$$
l u+m v+n w=0,
$$

where $l, m, n$ are the direction cosines of the outward-drawn normal. Averaging the terms in this equation and assuming that $\bar{u}, \vec{v}, \bar{w}$ vary slowly in an interval of length $r$, we have for all points on the fixed surface

$$
l \bar{u}+m \bar{v}+n \bar{w}=0,
$$

which is the desired boundary condition for a fixed solid wall. If the wall is smooth, the argument is repeated, replacing the height of the asperities by the thickness of the laminar boundary layer.

Next we consider a boundary condition which applies to the free surface. Let $F(x, y, z, t)=0$ be the equation of the free surface. As before in the case of the velocity components and the pressure (eq 1), the surface is considered to fluctuate rapidly and irregularly about a mean value, and we write

$$
F=\bar{F}+F^{\prime},
$$

where $\vec{F}$ is the mean value of $F$, and $F^{\prime}$ is the fluctuation whose temporal mean value $\overline{F^{\prime \prime}}$ is zero.

Since particles on the surface remain there, we have the condition

$$
\frac{\partial F}{\partial t}+u \frac{\partial F}{\partial x}+v_{s} \frac{\partial F}{\partial y}+w_{s} \frac{\partial F}{\partial z}=0
$$

where $u_{s}, v_{s}, w_{s}$ are the velocity components at the free surface. If we replace $u_{s}, v_{s}, w_{s}$ by expressions obtained from eq 1 and $F$ by its value from eq 19 and average the terms, the result is

$$
\frac{\partial \bar{F}}{\partial t}+\bar{u}_{s} \frac{\partial \bar{F}}{\partial x}+\overline{v_{s}} \frac{\partial \bar{F}}{\partial y}+\bar{w}_{s} \frac{\partial \bar{F}}{\partial z}=0
$$

provided we neglect the terms $\overline{u_{s}^{\prime}\left(\partial F^{\prime} / \partial x\right)}, \ldots$ These products 
may be ignored, since either the correlation between $u_{s}^{\prime}, \partial F^{\prime} / \partial x$, etc., is zero or the term is small in comparison with those retained. On the basis of this assumption, the above equation is a boundary condition obtaining at the free surface of turbulent flow.

In order to simplify the notation from this point on, $u, v, w, p$, etc., will be written for $\bar{u}, \bar{v}, \bar{w}, \bar{p}$, etc. Any further use of the bar to indicate an average will be in the macroscopic, rather than the microscopic, sense.

\section{EQUATION OF CONTINUITY FOR MEAN FLOW IN CHANNELS}

Up until this point we have derived equations which are valid for turbulent flow in general. We now derive an equation which applies to any tube of flow with a cross section which varies both in space and in time. This tube is assumed not to close on itself, and since it is a mathematical representation of a flowing channel, it is drawn as such in figure 2. The cross-sectional area of the channel, normal to $x$, will be represented by $A=A(x, t)$, the average value of $u$ over the cross section by $U=U(x, t)$.

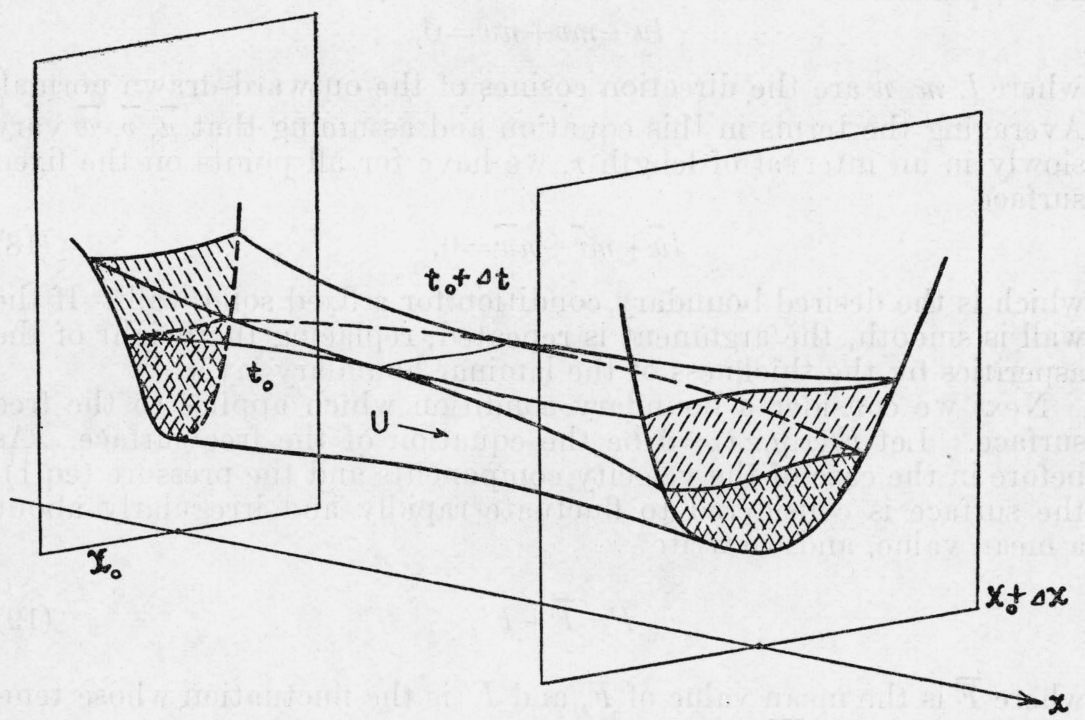

Figure 2.-Surface configurations at two different times in an open channel.

The positive sense of $x$ is chosen so as to make $U$ positive. The actual direction of $x$ is arbitrary, but for convenience it should approximate the direction of the channel.

The flow into the stretch $\Delta x$ during the time $\Delta t$ is

$$
\int_{t_{0}}^{t_{0}+\Delta t} U\left(x_{0}, t\right) A\left(x_{0}, t\right) d t
$$

while the flow out of the same stretch is

$$
\int_{t_{0}}^{t_{0}+\Delta t} U\left(x_{0}+\Delta x, t\right) A\left(x_{0}+\Delta x, t\right) d t .
$$


Furthermore, the volume of liquid contained within the stretch at time $t_{0}$ is

$$
\int_{x_{0}}^{x_{0}+\Delta x} A\left(x, t_{0}\right) d x
$$

while the volume in the stretch at time $t_{0}+\Delta t$ is

$$
\int_{x_{0}}^{x_{0}+\Delta x} A\left(x, t_{0}+\Delta t\right) d x
$$

Since the fluid is incompressible, net outflow plus increase of volume equals zero, or

$$
\begin{aligned}
\int_{t_{0}}^{t_{0}+\Delta t}\left[U\left(x_{0}+\Delta x, t\right) A\left(x_{0}+\Delta x, t\right)-U\right. & \left.\left(x_{0}, t\right) A\left(x_{0}, t\right)\right] d t \\
& +\int_{x_{0}}^{x_{0}+\Delta x}\left[A\left(x, t_{0}+\Delta t\right)-A\left(x, t_{0}\right)\right] d x=0 .
\end{aligned}
$$

If we apply the theorem of the mean for integrals, divide by $\Delta x \Delta t$ and then let $\Delta x$ and $\Delta t$ approach zero, we obtain

$$
\frac{\partial}{\partial x}(U A)+\frac{\partial A}{\partial t}=0
$$

which is the equation of continuity for mean flow in channels. Since no assumptions have been made regarding the shape of the channel, this equation applies to open channels in all generality.

\section{FIRST-ORDER THEORY OF WAVE PROPAGATION}

\section{THE EQUATION OF MEAN FLOW IN PRISMATIC CHANNELS}

Consider a prismatic channel and select as the origin of a righthanded system of Cartesian coordinates the lowest point of any convenient cross section (see fig. 3). Take the $x$-axis as the line parallel to the channel (not necessarily horizontal), with positive sense in the

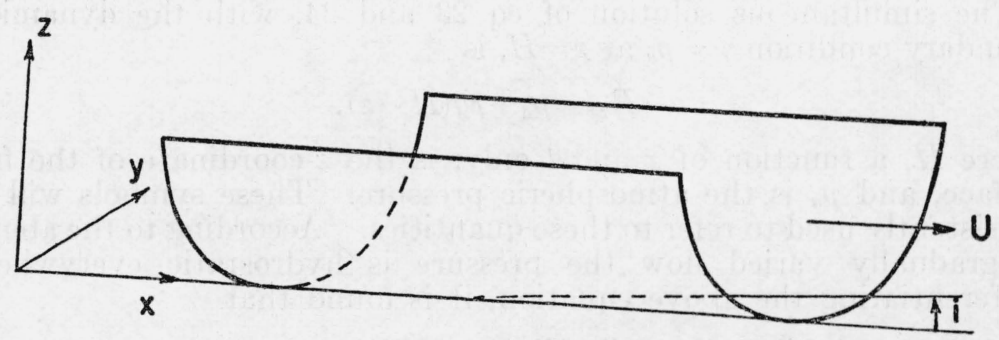

FIGURE 3.-System of coordinates in a prismatic channel.

direction of flow, the $y$-axis as the horizontal line, and the $z$-axis positive upward (not necessarily vertical). The channel slope, $i$, is the tangent of the angle $\theta$ from the positive direction of the $x$-axis to the horizontal, measured in the counterclockwise direction, viewed in the direction of increasing $y$; in other words, a positive slope is downhill. These conventions will be adhered to throughout the paper. 
We assume that the angle of slope of the channel is so small that $\sin \theta$ is sensibly equal to $\tan \theta$ and $\cos \theta$ to unity, hence $X$ and $Z$ in eq 10 are $g i$ and $-g$, respectively.

Again, in order to simplify the three equations of motion, eq 10 , we make two distinct sets of assumptions, the first involving the terms on the left-hand side and the second involving the terms on the right-hand side. First, we assume that the second derivatives of the cross section, $A$, and of the mean velocity, $U$, and the squares and products of their first derivatives, may be neglected in comparison with the first derivatives. Such a flow is referred to by Boussinesq as gradually varied [3, p. 242]. In general, this requires that the sides of the channel be not much flatter than a 1:1 slope, that the slope of the wave profile be small compared to unity, and that the curvature of the wave profile be small compared to $(1 / U)(\partial U / \partial x)$ or $\left(1 / U^{2}\right)$ $(\partial U / \partial t)$. As a result of these restrictions, $d v / d t$ and $d w / d t$ are small with respect to $d u / d t$, and the left-hand sides of the last two of eq 10 vanish.

Secondly, we make certain assumptions concerning the Reynolds stresses: (1) $\overline{u^{\prime} u^{\prime}}=\overline{v^{\prime} v^{\prime}}=\overline{w^{\prime} w^{\prime}}$ hence $R_{x x}=R_{y y}=R_{z z}$; (2) since $u$ is the predominant velocity component, the derivative of the correlation between the fluctuations $v^{\prime}, w^{\prime}$ may be neglected in comparison with the derivatives of the correlations between $u^{\prime}, v^{\prime}$, and $u^{\prime}, w^{\prime}$, which is to say, that the derivatives of $R_{z y}$ may be neglected; (3) since the flow is gradually varied, the $x$-derivatives of $R_{x y}$ and $R_{x z}$ may be neglected.

On the basis of these two sets of assumptions, then, eq 10 reduce to

$$
\begin{gathered}
\frac{\partial u}{\partial t}+u \frac{\partial u}{\partial x}+v \frac{\partial u}{\partial y}+w \frac{\partial u}{\partial z}=g i-\frac{1}{\rho} \frac{\partial}{\partial x}\left(p-R_{x x}\right)+\frac{1}{\rho} \frac{\partial}{\partial y} R_{y x}+\frac{1}{\rho} \frac{\partial}{\partial z} R_{z x} \\
0=\frac{\partial}{\partial y}\left(p-R_{x x}\right)
\end{gathered}
$$

and

$$
0=\frac{1}{\rho} \frac{\partial}{\partial z}\left(p-R_{x x}\right)+g .
$$

The simultaneous solution of eq 23 and 24, with the dynamical boundary condition $p=p_{a}$ at $z=H$, is

$$
p-R_{x x}=p_{a}+\rho g(H-z),
$$

where $H$, a function of $x$ and $t$ only, is the $z$-coordinate of the free surface, and $p_{a}$ is the atmospheric pressure. These symbols will be consistently used to refer to these quantities. According to the above, in gradually varied flow the pressure is hydrostatic everywhere. Differentiating the above equation, it is found that

$$
\frac{\partial\left(p-R_{x x}\right)}{\partial x}=\rho g \frac{\partial H}{\partial x} .
$$

Each term in eq 22 is to be integrated over the cross section $A$ at $x$ In this way, the resulting equation of motion will involve only the mean flow, $U$, the cross-sectional area, $A$, and certain other quantities to be given later. We thus eliminate the variables $y$ and $z$ from consideration, since all of the quantities occurring in the final result will 
be functions of $x$ and $t$ only. Such an equation is called a hydraulic equation, and bears the same relation to circuit equations in electricity as the general equations 10 do to the electromagnetic field equations.

Let us first evaluate the integrals arising from the terms in the lefthand side. The mean flow, $U$, in a cross section, is implicitly defined by

$$
U A=\int_{A} u d A .
$$

We write the above as a double integral

$$
U A=\int_{0}^{H} d z \int_{-y_{1}}^{y_{2}} u d y
$$

where $y_{2}$ and $-y_{1}$ are the $y$-coordinates of the two points on the wetted perimeter having the common distance $z$ from the $x, y$-plane (see fig. 4). Obviously, $y_{2}$ and $-y_{1}$ are functions of $z$ only, for a given prismatic channel configuration. Differentiating both sides of the above equation with respect to $t$, we obtain

$$
\frac{\partial(U A)}{\partial t}=\int_{A} \frac{\partial u}{\partial t} d A+\frac{\partial H}{\partial t} \int_{-b_{1}}^{b_{2}} u_{s} d y
$$

where $b_{2}$ and $-b_{1}$ are the $y$-coordinates of the two points on the wetted perimeter at the distance $H$ from the $x, y$-plane.

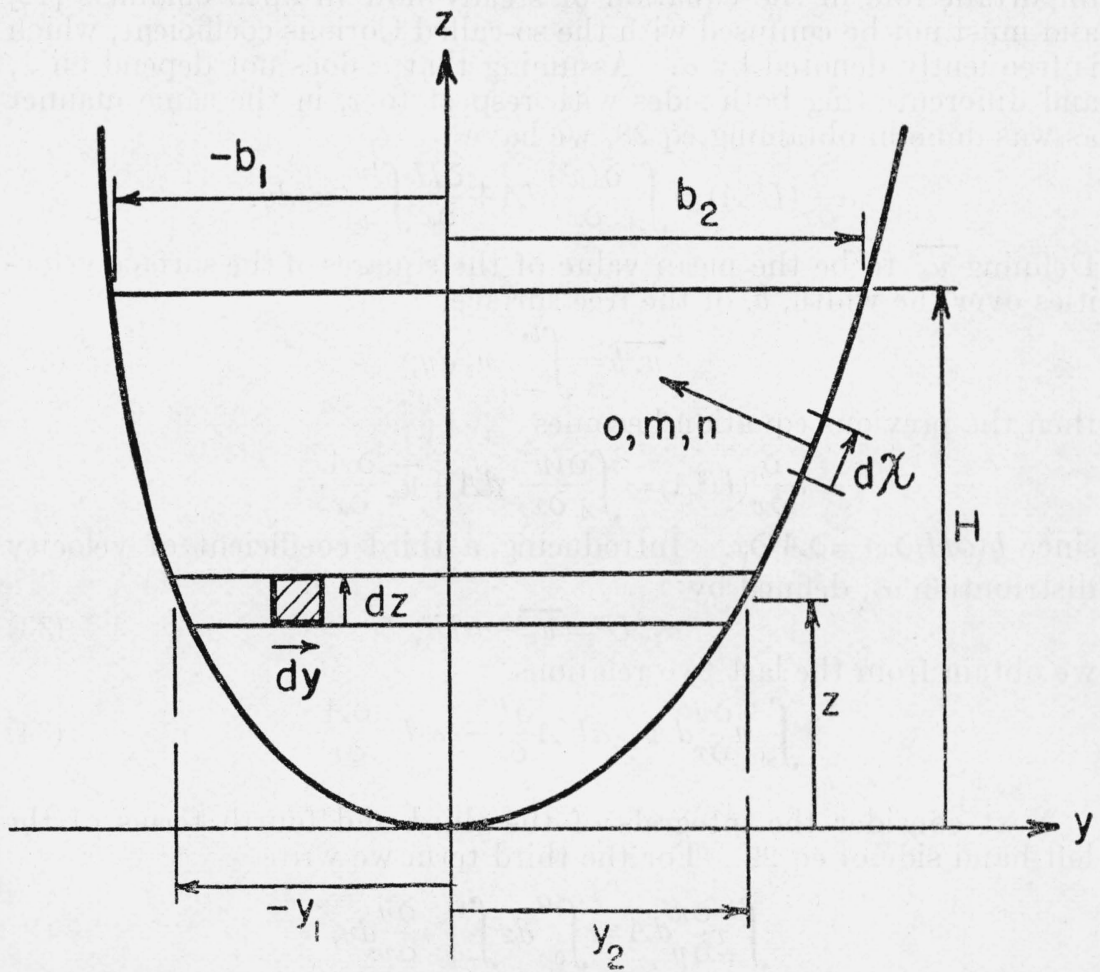

FIGURE 4.-Double integration with respect to $y$ and $z$; cross section, looking upstream • $524586-43-4$ 
We now define $\bar{u}_{s}$ to be the mean value of the surface velocities, $u_{s}$, over the width $b=b_{2}+b_{1}$ of the free surface:

$$
\overline{u_{s}} b=\int_{-b_{1}}^{b_{2}} u_{s} d y
$$

and the next to the last equation becomes

$$
\frac{\partial}{\partial t}(U A)=\int_{A} \frac{\partial u}{\partial t} d A+\bar{u}_{s} \frac{\partial A}{\partial t},
$$

since $b(\partial H / \partial t)=\partial A / \partial t$. Introducing the velocity coefficient $\alpha_{1}$, implicitly defined by

eq 28 may be written

$$
\alpha_{1} U=\bar{u}_{s}-U,
$$

$$
\int \frac{\partial u}{\partial t} d A=A \frac{\partial U}{\partial t}-\alpha_{1} U \frac{\partial A}{\partial t}
$$

We now turn our attention to the second term on the left of eq 22 . We define another coefficient of velocity distribution $\alpha$, as the ratio of the mean of the square of velocity to the square of its mean, both means being taken over the cross section, thus

$$
\alpha U^{2} A=\int_{A} u^{2} d A
$$

This coefficient is the only one which appears in the final equation, and was originally introduced by Boussinesq. It also plays an important role in the equation of steady flow in open channels [12] and must not be confused with the so-called Coriolis coefficient, which is frequently denoted by $\alpha$. Assuming that $\alpha$ does not depend on $x$, and differentiating both sides with respect to $x$, in the same manner as was done in obtaining eq 28 , we have

$$
\alpha \frac{\partial}{\partial x}\left(U^{2} A\right)=\int_{A} \frac{\partial\left(u^{2}\right)}{\partial x} d A+\frac{\partial H}{\partial x} \int_{-b_{1}}^{b_{2}} u_{s}^{2} d y
$$

Defining $\overrightarrow{u_{\mathrm{s}}}$ to be the mean value of the squares of the surface velocities over the width, $b$, of the free surface,

then the previous equation becomes

$$
\overline{u_{s}^{2}} b=\int_{-b_{1}}^{b_{2}} u_{s}^{2} d y
$$

$$
\alpha \frac{\partial}{\partial x}\left(U^{2} A\right)=\int \frac{\partial\left(u^{2}\right)}{\partial x} d A+\overline{u_{s}^{2}} \frac{\partial A}{\partial x}
$$

since $b(\partial H / \partial x)=\partial A / \partial x$. Introducing a third coefficient of velocity distribution $\alpha_{2}$, defined by

$$
\alpha_{2} 2 U^{2}=\overline{u_{s}^{2}}-\alpha U^{2},
$$

we obtain from the last two relations,

$$
\int_{A} u \frac{\partial u}{\partial x} d A=\alpha U A \frac{\partial U}{\partial x}-\alpha_{2} U^{2} \frac{\partial A}{\partial x} \text {. }
$$

Next consider the integrals of the third and fourth terms of the left-hand side of eq 22 . For the third term we write

$$
\int_{A} v \frac{\partial u}{\partial y} d A=\int_{0}^{H} d z \int_{-y_{1}}^{y_{2}} v \frac{\partial u}{\partial y} d y
$$


where the notation is the same as that used in the above two integrals (see fig. 4). The integral with respect to $y$ is integrated by parts, obtaining

$$
\int_{A} v \frac{\partial u}{\partial y} d A=\int_{0}^{H}\left[\left(u_{0} v_{0}\right)_{2}-\left(u_{0} v_{0}\right)_{1}\right] d z-\int_{A} u \frac{\partial v}{\partial y} d A,
$$

where $v_{0}$ and $u_{0}$ are the velocity components at the wetted perimeter and the subscripts 1 and 2 indicate the two sides of the channel, that is, the points $\left(-y_{1}, z\right)$ and $\left(y_{2}, z\right)$, respectively.

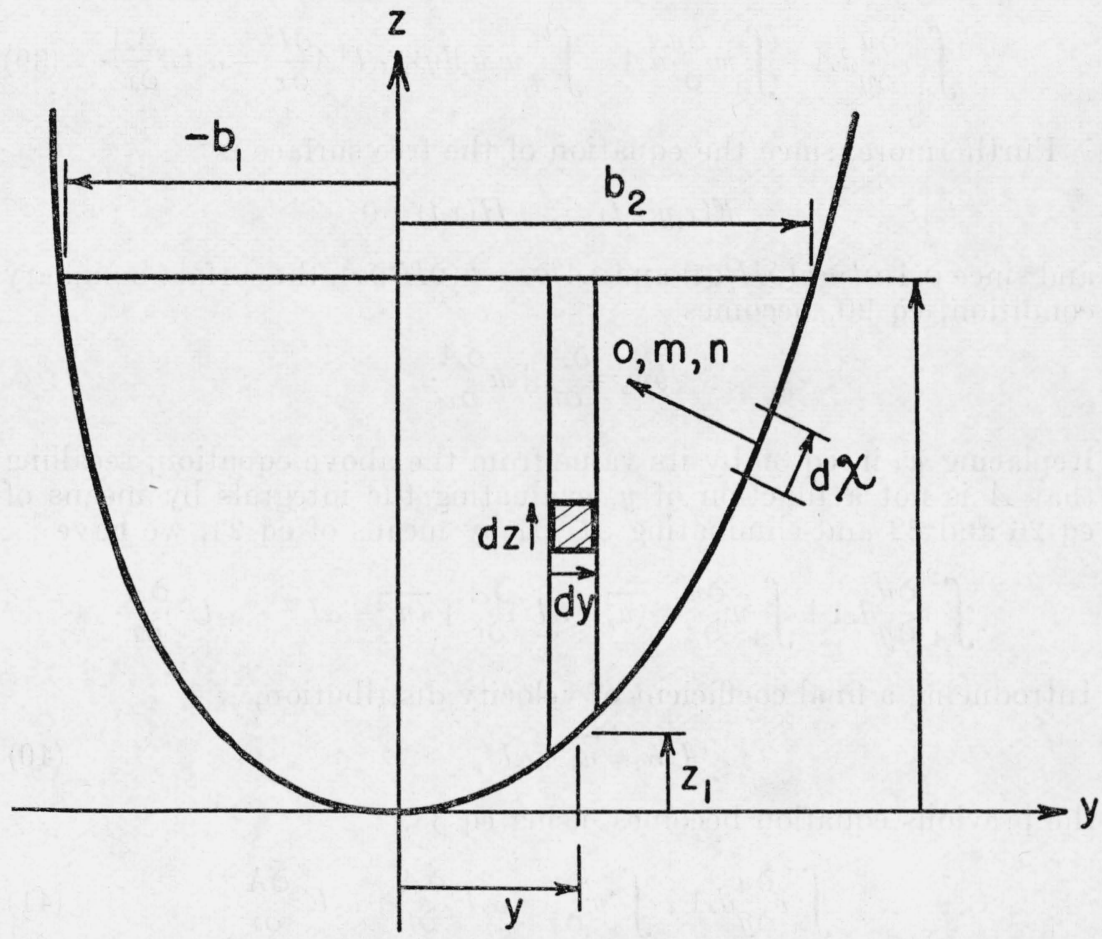

Figure 5.-Double integration with respect to $z$ and $y$; cross section, looking upstream.

Again, for the fourth term

$$
\int_{A} w \frac{\partial u}{\partial z} d A=\int_{-b_{1}}^{b_{2}} d y \int_{z_{1}}^{H} w \frac{\partial u}{\partial z} d z
$$

where $z_{1}$ is the $z$-coordinate of a point on the wetted perimeter at the distance $y$ from the $x, z$-plane (see fig. 5). This time the integral with respect to $z$ is integrated by parts, obtaining

$$
\int_{A} w \frac{\partial u}{\partial z} d A=\int_{-b_{1}}^{b_{2}}\left(w_{s} u_{s}-w_{0} u_{0}\right) d y-\int u_{\partial z}^{\partial} d A
$$

where $w_{0}, u_{0}$ are now evaluated at the point $\left(y, z_{1}\right)$.

If $d \chi$ denotes an elementary arc of the wetted perimeter, $\chi$, measured in the counterclockwise direction, and the direction cosines of the inward-drawn normal are $0, m, n$ (figs. 4 and 5) then $d y=n d x$ and $d z=-m d x$, and 35 and 36 become 


$$
\int_{A} v \frac{\partial u}{\partial y} d A=-\int_{\chi} u_{0} v_{0} m d \chi-\int_{A} u \frac{\partial v}{\partial y} d A
$$

and

$$
\int_{A} w \frac{\partial u}{\partial t} d A=\int_{-b_{1}}^{b_{2}} w_{s} u_{s} d y-\int_{\chi} w_{0} u_{0} n d \chi-\int_{A} u \frac{\partial w}{\partial z} d A .
$$

We add these two equations together, noting that as a result of eq 18 the integrals with respect to $\chi$ vanish, and combine the integrals in the extreme right by means of eq 8 and 34 obtaining

$$
\int_{A} v^{\frac{\partial u}{\partial y}} d A+\int_{A} w \frac{\partial u}{\partial z} d A=\int_{-b_{1}}^{b_{2}} w_{3} u_{s} d y+\alpha U A \frac{\partial U}{\partial x}-\alpha_{2} U^{2} \frac{\partial A}{\partial x} .
$$

Furthermore, since the equation of the free surface is

$$
F(x, y, z, t)=z-H(x, t)=0,
$$

and since $\partial A / \partial t=b(\partial H / \partial t)$ and $\partial A / \partial x=b(\partial H / \partial x)$, the surface boundary condition, eq 20 , becomes

$$
b w_{s}=\frac{\partial A}{\partial t}+u_{s} \frac{\partial A}{\partial x}
$$

Replacing $w_{s}$ in eq 39 by its value from the above equation, recalling that $A$ is not a function of $y$, evaluating the integrals by means of eq 26 and 32 and eliminating $\partial U / \partial x$ by means of eq 21 , we have

$$
\left.\left.\int_{A} v \frac{\partial u}{\partial y} d A+\int_{A} w \frac{\partial u}{\partial z}=\overline{\left(u_{s}\right.}-\alpha U\right) \frac{\partial A}{\partial t}+\overline{\left(u_{s}^{2}\right.}-\alpha U^{2}-\alpha_{2} U^{2}\right) \frac{\partial A}{\partial x} .
$$

Introducing a final coefficient of velocity distribution,

$$
U \alpha_{3}=\overline{u_{s}}-\alpha U \text {, }
$$

the previous equation becomes, using eq 33 ,

$$
\int_{A} v \frac{\partial u}{\partial y} d A+\int_{A} w \frac{\partial u}{\partial z}=\alpha_{3} U \frac{\partial A}{\partial t}+\alpha_{2} U^{2} \frac{\partial A}{\partial x} .
$$

We have now succeeded in evaluating all the terms on the left of eq 22 in terms of functions of $x$ and $t$ only, and proceed to the terms on the right. In view of eq 25 , the first two terms become merely

$$
g i \int_{A} d A-\frac{1}{\rho} \int_{A} \frac{\partial}{\partial x}\left(p-R_{x x}\right) d A=g i A-g A \frac{\partial H}{\partial x},
$$

since $H$ is not a function of $y$ or $z$.

The integral of the last two terms in eq 22 is transformed by Green's theorem into a line integral around the periphery of the cross section:

$$
\frac{1}{\rho} \int_{A}\left[\frac{\partial}{\partial y} R_{y x}+\frac{\partial}{\partial z} R_{z x}\right] d A=\frac{1}{\rho} \oint R_{\nu x} d z-R_{z x} d y .
$$


Expressing the line integral in terms of $\chi$ as was done for eq 37 and 38,

$$
\frac{1}{\rho} \int_{A}\left[\frac{\partial}{\partial y} R_{y x}+\frac{\partial}{\partial z} R_{z x}\right] d A=-\frac{1}{\rho} \oint\left[m R_{y x}+n R_{z x}\right] d \chi .
$$

Comparison with eq 14 and 15 shows that the integrand of the line integral is the $x$-component of the force which the fluid exerts on the channel walls and the free surface. If we denote this shearing force by $\tau$, and assume that the shear on the free surface is zero, and define $\tau_{0}$ to be the mean shear of the fluid on the channel walls and bottom

then we have

$$
\tau_{0}=\frac{1}{\chi} \int_{\chi} \tau d \chi,
$$

$$
\frac{1}{\rho} \int_{A}\left[\frac{\partial}{\partial y} R_{y x}+\frac{\partial}{\partial z} R_{z x}\right] d A=-\frac{\tau_{0} \chi}{\rho}
$$

Our choice of axes makes $\tau_{0}$ positive, since $\tau_{0}$ has the same direction as $U$. We now define $\lambda$ the channel coefficient of friction,

$$
\tau_{0}=\lambda \frac{\rho U^{2}}{2},
$$

and eq 44 becomes

$$
\frac{1}{\rho} \int\left[\frac{\partial}{\partial i} R_{i x}+\frac{\partial}{\partial z} R_{z x}\right] d A=-\frac{\lambda U^{2} \chi}{2} .
$$

Collecting the results of eq $30,34,41,42,46$, and dividing them by $A$, the desired integral of eq 22 is

$$
g^{\partial H}+\frac{\partial U}{\partial t}+\alpha U \frac{\partial U}{\partial x}+(1-\alpha) \frac{U \partial A}{A} \frac{\partial}{\partial t}=g i-\frac{\lambda U^{2}}{2 R},
$$

where $R=A / \chi$ is the hydraulic radius of the channel. This is the desired hydraulic equation of motion for gradually varied unsteady flow in prismatic channels. It will be noted that only one coefficient of velocity distribution enters into the final equation, the one related to the distribution of the squares of velocities in a cross section.

This equation was derived on the basis of the assumption that the flow was turbulent, which is the case usually met with in practice. Nevertheless, the equation also applies to viscous laminar flow, since the dynamical equations of motion, eq 13, are the same for both types of flow, except that in laminar flow the quantities $p_{x x}, \ldots$., are replaced by $S_{x x}, \ldots$, rather than by $R_{x x}, \ldots$ It is tacitly assumed that the flow pattern is such that the assumptions made concerning the Reynolds stresses in deriving eq 22, 23, 24 are also true for the viscous stresses. Finally, in laminar flow $\alpha$ and $\lambda$ become quantities that may be calculated rather than ones that must be experimentally determined.

2. COEFFICIENT OF RESISTANCE AS A FUNCTION OF $H$

If it is assumed that the coefficient $\lambda$ defined by eq 45 is a function of $H$ only, and is therefore independent of $\partial U / \partial t$ and $\partial U / \partial x$, then $\lambda$ can 
be evaluated from the ordinary parameters of uniform flow. Since, when the flow is uniform, $U$ and $H$ are constants, we have from eq 47

or

$$
g i=\frac{\lambda U_{0}^{2}}{2 R_{0}}
$$

$$
\frac{U_{0}}{\sqrt{g i R_{0}}}=\sqrt{\frac{2}{\lambda}}
$$

where $U_{\mathrm{o}}$ and $R_{\mathrm{o}}$, respectively, are the mean velocity and hydraulic radius, for uniform flow.

We assume further that the propagation of the waves is restricted to that type of turbulent flow in which the friction is determined by the surface asperities independently of the Reynolds number. The resistance law is then represented adequately by Manning's formula in the following form [5, eq 61, 80]:

$$
\frac{U_{0}}{\sqrt{g i R_{0}}}=8.12\left(\frac{R_{0}}{k_{s}}\right)^{1 / 6}
$$

where $k_{s}$ is the equivalent sand roughness [5, p. 713] of the channel surfaces.

Equating the right-hand members of eq 48 and 49, we obtain

$$
\lambda=0.0303\left(\frac{k_{s}}{R_{0}}\right)^{1 / 3}
$$

Differentiating with respect to $H_{0}$, the value of $H$ for undisturbed flow, we have

$$
\frac{d \lambda}{d H_{0}}=-\frac{\lambda}{3 R_{0}} \frac{d R_{0}}{d H_{0}}
$$

since $R_{0}$ is a function of $H_{0}$. For a wide rectangular channel, $R=H$, and

$$
\frac{d \lambda}{d H_{0}}=-\frac{\lambda}{3 H_{0}}
$$

\section{VELOCITY OF PROPAGATION OF SHORT WAVES WITH NEGLIGIBLE CURVATURE}

Assuming an initial condition of uniform flow in a very wide rectangular channel, let $H_{0}$ be the depth of the liquid and $U_{0}$ be the mean velocity of flow in a cross-section. If the flow is disturbed, this can be expressed by writing:

and

$$
\left.\begin{array}{l}
H(x, t)=H_{0}+h(x, t), \\
U(x, t)=U_{0}+u(x, t),
\end{array}\right\}
$$

for the depth and for the mean velocity. The quantity $u$ will, in general, henceforth signify the variation from $U_{0}$, the undisturbed value of $U$, and $h$ the variation from $H_{0}$, the undisturbed value of $H$. We replace $H$ and $U$ in eq 47 by their values from eq 52 . Since the 
channel is rectangular, $A$ is a linear function of $H$; since it is wide, $R=H$; and $H_{0}$ and $U_{0}$ are constants, hence eq 47 and 21 become

$$
\begin{gathered}
g \frac{\partial h}{\partial x}+\frac{\partial u}{\partial t}+\alpha\left(U_{0}+u\right) \frac{\partial u}{\partial x}+(1-\alpha) \frac{U_{0}+u}{H_{0}+h} \frac{\partial h}{\partial t}=g i-\frac{\lambda\left(U_{0}+u\right)^{2}}{2\left(H_{0}+h\right)}, \\
\frac{\partial h}{\partial t}+\left(H_{0}+h\right) \frac{\partial u}{\partial x}+\left(U_{0}+u\right) \frac{\partial h}{\partial x}=0 .
\end{gathered}
$$

We now introduce two restrictions of a different kind. First, we assume that $\alpha$ is very nearly equal to unity and hence the term containing 1- $\alpha$ may be neglected. Secondly, we assume that

$$
\begin{array}{cl}
\text { (I) } & u \frac{\partial u}{\partial x} \ll \frac{\partial u}{\partial t}, \\
\text { (II) } & g i-\frac{\lambda U^{2}}{2 H} \ll \frac{\partial u}{\partial t}, \\
\text { (III) } \quad u \frac{\partial h}{\partial x}+h \frac{\partial u}{\partial x} \ll \frac{\partial h}{\partial t} .
\end{array}
$$

On this basis eq 53 and 54 reduce to

$$
\begin{array}{r}
g_{\partial x} \frac{\partial h}{\partial t}+\frac{\partial u}{\partial t}+U_{0} \frac{\partial u}{\partial x}=0 \\
\frac{\partial h}{\partial t}+H_{0} \frac{\partial u}{\partial x}+U_{0} \frac{\partial h}{\partial x}=0 .
\end{array}
$$

We differentiate eq 58 with respect to $x$ and eliminate the terms containing $u$ by means of eq 59. This gives

$$
\frac{\partial^{2} h}{\partial t^{2}}+2 U_{0}{ }^{2} \frac{\partial^{2} h}{\partial x \partial t}+\left(U_{0}^{2}-g H_{0}\right) \frac{\partial^{2} h}{\partial x^{2}}=0 .
$$

A particular solution of this is

$$
h=F\left(x-\omega_{0} t\right),
$$

where $F$ is an arbitrary function, provided $\omega_{0}$ is a root of

$$
\omega_{0}^{2}-2 U_{0} \omega_{0}+U_{0}^{2}-g H_{0}=0 .
$$

The two roots are

$$
\omega_{0}=U_{0} \pm \sqrt{g H_{0}},
$$

and it is evident upon inspecting eq 61 that the wave is propagated without change of form with the celerity, or velocity of propagation, $\omega_{0}$ in the positive $x$-direction, since at time $t+\left(\Delta x / \omega_{0}\right)$, the wave profile is displaced by $\Delta x$ from its position at time $t$. When an observer is moving with the current, that is, moving with the velocity $U_{0}$, a wave may be moving either downstream or upstream with respect to the observer. In the first case, $U_{0}<\omega_{0}$, and the wave is descending; in the second case, $U_{0}>\omega_{0}$, and the wave is ascending. Accordingly, the larger root of $\omega_{0}$ gives the celerity of a descending wave and the smaller root the celerity of an ascending wave. In all formulas containing an ambiguous sign the upper sign will pertain to descending waves and the lower sign to ascending waves. 
480 Journal of Research of the National Bureau of Standards

It is now a simple matter to determine the function $u$. From eq 61 , we have

$$
\frac{\partial h}{\partial t}=-\omega_{0} \frac{\partial h}{\partial x} .
$$

Substituting this value of $\partial h / \partial t$ in eq 59 , it becomes

$$
\frac{\partial u}{\partial x}+\frac{U_{0}-\omega_{0}}{H_{0}} \frac{\partial h}{\partial x}=0
$$

whose integral is

$$
u+\frac{U_{0}-\omega_{0}}{H_{0}} h+f(t)=0 .
$$

Since for any time $t$, at $h=0, u=0$, then since the liquid is undisturbed at infinity $f(t) \equiv 0$, and

$$
u=\left(\omega_{0}-U_{0}\right) \frac{h}{H_{0}} ;
$$

or in view of eq 62

$$
u= \pm \sqrt{g H_{0}} \frac{h}{\bar{H}_{0}}
$$

which states that the excess of the mean velocity of the particles in a cross section under the wave is proportional to the wave height $h$.

It will be noted that the above equation for the celerity of waves, eq 62 , is the same as that developed for the motion of irrotational waves in still water [1, eq 56], except that a constant velocity of flow, $U_{0}$, is superposed on the wave velocities relative to the water, $\sqrt{g H_{0}}$. This would appear to indicate that the friction due to turbulence which is inherent in the undisturbed flow, $U_{0}$, has no effect on the propagation of waves. In general, the effect of turbulence cannot be neglected, but in this case the assumptions made in deriving eq 62 restrict the size of the wave in such a way that the effect of turbulence is negligible.

The physical significance of these mathematical assumptions will now be investigated. Consider first

$$
u \frac{\partial h}{\partial x}+h \frac{\partial u}{\partial x} \ll \frac{\partial h}{\partial t}
$$

Replacing $u$ by its value from eq 64 , and $\partial h / \partial t$ by its value from eq 63 , we have

$$
\frac{\hbar}{H_{0}} \ll \frac{1}{2}\left(\frac{\omega_{0}}{U_{0}-\omega_{0}}\right) .
$$

Consider secondly

(I) $\quad u \frac{\partial u}{\partial x} \ll \frac{\partial u}{\partial t} \cdot$

Using the same relations as before, we have

$$
\frac{h}{H_{0}} \ll \frac{\omega_{0}}{U_{0}-\omega_{0}} .
$$

Obviously, the first condition is sufficient for the second. 
Both of these restrictions have no bearing on the fact that the undisturbed flow is controlled by friction. They are necessary but are not sufficient conditions for a wave to move without change of form. A more significant restriction arises from the assumption that the right-hand member of eq 53 is negligible:

$$
\text { (II) } \quad g i-\frac{\lambda\left(U_{0}+u\right)^{2}}{2\left(H_{0}+h\right)} \ll \frac{\partial u}{\partial t} \text {. }
$$

Expressing the left-hand side in terms of $u / \mathrm{U}_{0}$ and $h / H_{0}$, and replacing $\lambda$ by its value from eq 48 , we obtain, since $R_{0}=\mathrm{H}_{0}$,

$$
\frac{g i \frac{h}{H_{0}}\left(1-2 \frac{u H_{0}}{U_{0} h}-\left(\frac{u}{U_{0}}\right)^{2} \frac{H_{0}}{h}\right)}{1+\frac{h}{H_{0}}} \ll \frac{\partial u}{\partial t} .
$$

Eliminating $u$ via eq 64 , and transforming the right-hand side by means of eq 63 , we have

$$
\frac{g i H_{0}\left(3-\frac{2 \omega_{0}}{U_{0}}-\left(\frac{\omega_{0}}{U_{0}}-1\right)^{2} \frac{h}{H_{0}}\right)}{-\omega_{0}\left(\omega_{0}-U_{0}\right)\left(1+\frac{h}{H_{0}}\right)}\left(\frac{h}{H_{0}}\right) \ll \frac{\partial h}{\partial x} .
$$

In contrast to conditions (I) and (II), where we obtained an inequality comparing $h / \mathrm{H}_{0}$ with a constant term which is determined by the characteristics of the channel, we find in this case that both sides of the inequality are functions of $h$. We are also confronted with the fact that $\partial h / \partial x$ generally vanishes at the apex of the wave profile, and hence the inequality cannot be satisfied at that point even if the left-hand member also vanishes.

In order to arrive at a workable criterion for determining whether the theory is applicable, we integrate both sides of the inequality with respect to $x$, thus obtaining an average condition:

$$
\frac{i g H_{0}}{-\omega_{0}\left(\omega_{0}-U_{0}\right)} \int \frac{3-2 \frac{\omega_{0}}{U_{0}}-\left(\frac{\omega_{0}}{U_{0}}-1\right)^{2} \frac{h}{H_{0}}}{1+\frac{h}{H_{0}}}\left(\frac{h}{H_{0}}\right)\left|d x \ll \int\right| \frac{\partial h}{\partial x} \mid d x .
$$

It is not feasible to integrate the left-hand side of this inequality unless the function $h(x, t)$ is given. It is therefore necessary to assume that

$$
\text { (IV) } \quad\left(\frac{\omega_{0}}{U_{0}}-1\right)^{2} \frac{h}{H_{0}} \ll 3-\frac{2 \omega_{0}}{U_{0}},
$$

and

$$
\text { (V) } \quad \frac{h}{H_{0}} \ll 1 \text {, }
$$

and eq 67 reduces to

$$
B \int\left|\frac{h}{H_{0}}\right| d x \ll \int\left|\frac{\partial h}{\partial x}\right| d x
$$


where

$$
B=i g H_{0} \frac{2 \omega_{0}-3 U_{0}}{\omega_{0} U_{0}\left(\omega_{0}-U_{0}\right)} .
$$

If the wave profile is such that (IV) and (V) are not both satisfied, then eq 70 does not apply, and the integral on the left of eq 67 must be evaluated. Assuming that the wave profile has a unique maximum height $h_{m}$, that $\partial h / \partial x$ is everywhere finite, that $L$ is the length of the wave, and that $h$ is always positive, then eq 70 becomes

$$
\frac{L}{H_{0}} \ll \frac{2}{j B},
$$

where $j$ is a fraction such that the volume of the wave per unit width of channel above the undisturbed surface is $j h_{m} L$. This last inequality is the desired average condition, one which restricts the length of the wave. Waves fulfilling this condition will be referred to as short waves.

In addition to this restriction on the length of the wave, we still have the three conditions on the maximum height of the wave, expressed by eq 66,68 and 69 ; the first being derived from the original restriction (III), and the other two from the approximations used in obtaining eq 71. Each of the first two restrictions states that the maximum height of the wave must be small with respect to a constant determined by the channel parameters. For convenience we shall express these constants in terms of $U_{0} / \sqrt{g H_{0}}$.

For descending waves, $\omega_{0}=U_{0}+\sqrt{g H_{0}}$, and eq $66,68,69$ become

and

$$
\begin{array}{ll}
\text { (IIIa) } & \frac{h}{H_{0}} \ll-\frac{1}{2}\left(\frac{U_{0}}{\sqrt{g H_{0}}}+1\right), \\
\text { (IVa) } & \frac{h}{H_{0}} \ll \frac{U_{0}}{\sqrt{g H_{0}}}\left(\frac{U_{0}}{\sqrt{g H_{0}}}-2\right),
\end{array}
$$

$$
\frac{h}{H_{0}} \ll 1 \text {. }
$$

For ascending waves, $\omega_{0}=U_{0}-\sqrt{g H_{0}}$, and the same three equations become

and

$$
\begin{gathered}
\text { (IIIb) } \quad \frac{h}{H_{0}} \ll \frac{1}{2}\left(\frac{U_{0}}{\sqrt{g H_{0}}}-1\right) \\
\text { (IVb) } \quad \frac{h}{\bar{H}_{0}} \ll \frac{U_{0}}{g H_{0}}\left(\frac{U_{0}}{\sqrt{g H_{0}}}+2\right)
\end{gathered}
$$

$$
\frac{h}{H_{0}} \ll 1 \text {. }
$$

These relations are made more intelligible by means of figures 6 and 7 . The quantity $h / H_{0}$ must be small with respect to all of the quantities plotted in the figure, that is, small with respect to the 
least of them. It will be noted that the theory breaks down completely for ascending waves in water of critical depth, that is, it does not apply to the case of the hydraulic jump. The fact that it breaks

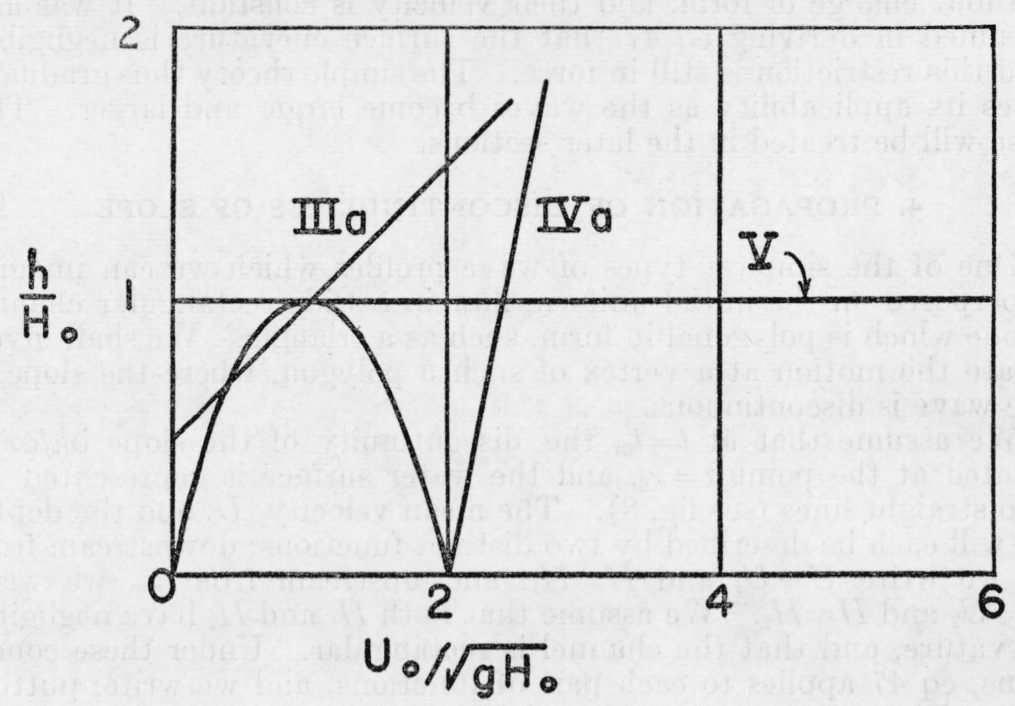

FIGURE 6.-Limitations on height of short descending waves moving without change of form.

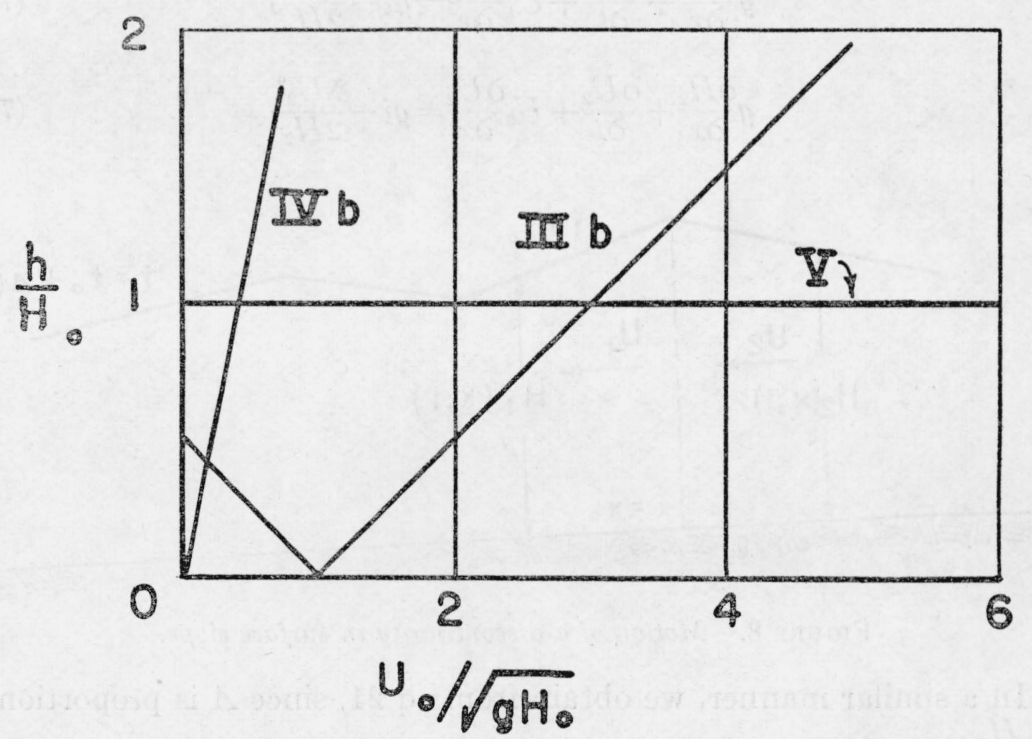

FIGURE 7.-Limitations on height of short ascending waves moving without change of form.

down for $U_{0} / \sqrt{g H_{0}}=2$ when the wave is descending is due merely to the approximation used in deriving eq 71 , and is not inherent in the theory. 
Our three original assumptions thus indicate that if a wave is short and of small height, and moving in a wide rectangular channel where the undisturbed current is controlled by friction, the waves move without change of form, and their velocity is constant. It was also assumed in deriving eq 47 that the surface curvature is negligible, and this restriction is still in force. The simple theory thus gradually loses its applicability as the waves become larger and larger. This case will be treated in the later sections.

\section{PROPAGATION OF DISCONTINUITIES OF SLOPE}

One of the simplest types of wave profiles which we can imagine superposed on the initial uniform flow in a wide rectangular channel is one which is polygonal in form, such as a triangle. We shall investigate the motion at a vertex of such a polygon, where the slope of the wave is discontinuous.

We assume that at $t=t_{0}$, the discontinuity of the slope $\partial h / \partial x$ is located at the point $x=x_{0}$, and the water surface is represented by two straight lines (see fig. 8). The mean velocity, $U$, and the depth, $H$, will each be described by two distinct functions; downstream from $x_{0}$, we write $U=U_{1}$ and $H=H_{1}$, and upstream from $x_{0}$, we write $U=U_{2}$ and $H=H_{2}$. We assume that both $H_{1}$ and $H_{2}$ have negligible curvature, and that the channel is rectangular. Under these conditions, eq 47 applies to each pair of functions, and we write, putting $\alpha$ equal to unity:

$$
\begin{aligned}
& g \frac{\partial H_{1}}{\partial x}+\frac{\partial U_{1}}{\partial t}+U_{1} \frac{\partial U_{1}}{\partial x}=g i-\frac{\lambda U_{1}^{2}}{2 H_{1}} \\
& g \frac{\partial H_{2}}{\partial x}+\frac{\partial U_{2}}{\partial t}+U_{2} \frac{\partial U_{2}}{\partial x}=g i-\frac{\lambda U_{2}^{2}}{2 H_{2}}
\end{aligned}
$$

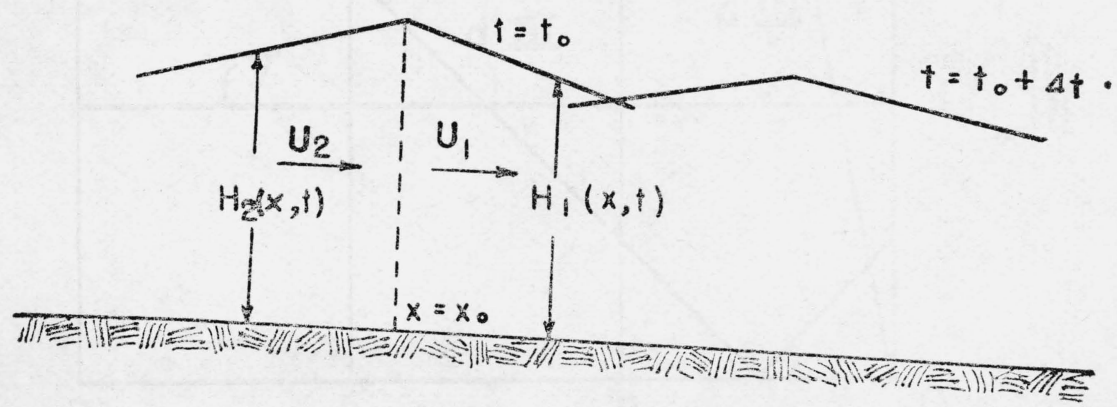

FIgURE 8.-Motion of a discontinuity in surface slope.

In a similar manner, we obtain from eq 21 , since $A$ is proportional to $H$,

$$
\begin{aligned}
& \frac{\partial H_{1}}{\partial t}+U_{1} \frac{\partial H_{1}}{\partial x}+H_{1} \frac{\partial U_{1}}{\partial x}=0 \\
& \frac{\partial H_{2}}{\partial t}+U_{2} \frac{\partial H_{2}}{\partial x}+H_{2} \frac{\partial U_{2}}{\partial x}=0 .
\end{aligned}
$$


Following Hadamard [13, p. 83], we introduce the following notation for the change in value of a derivative as the discontinuity is traversed:

$$
\frac{\partial H_{2}}{\partial x}-\frac{\partial H_{1}}{\partial x}=\left[\frac{\partial H}{\partial x}\right], \quad \frac{\partial U_{2}}{\partial x}-\frac{\partial U_{1}}{\partial x}=\left[\frac{\partial U}{\partial x}\right] .
$$

Subtracting eq 73 from eq 72 and eq 75 from eq 74 , we have, since $\mathrm{H}, \mathrm{U}$, and $\lambda$ are continuous,

$$
\begin{gathered}
g\left[\frac{\partial H}{\partial x}\right]+\left[\frac{\partial U}{\partial t}\right]+U\left[\frac{\partial U}{\partial x}\right]=0, \\
{\left[\frac{\partial H}{\partial t}\right]+U\left[\frac{\partial H}{\partial x}\right]+H\left[\frac{\partial U}{\partial x}\right]=0,}
\end{gathered}
$$

where $H$ and $U$ are evaluated at $x=x_{0}, t=t_{0}$. Since four discontinuities are involved, we need two more relations.

In figure 8 the displacement of the discontinuity during the time interval $\Delta t$ is indicated. If $\Delta U_{1}$ and $\Delta U_{2}$ are the changes in the mean velocities, then

$$
\begin{aligned}
& \Delta U_{1}=\frac{\partial U_{1}}{\partial x} \Delta x+\frac{\partial U_{1}}{\partial t} \Delta t \\
& \Delta U_{2}=\frac{\partial U_{2}}{\partial x} \Delta x+\frac{\partial U_{2}}{\partial t} \Delta t
\end{aligned}
$$

Similarly, if $\Delta H_{1}$ and $\Delta H_{2}$ are the changes in the height, then

$$
\begin{aligned}
& \Delta H_{1}=\frac{\partial H_{1}}{\partial x} \Delta x+\frac{\partial H_{1}}{\partial t} \Delta t, \\
& \Delta H_{2}=\frac{\partial H_{2}}{\partial x} \Delta x+\frac{\partial H_{2}}{\partial t} \Delta t .
\end{aligned}
$$

Subtracting eq 79 from 78 and 81 from 80 ,

$$
\begin{gathered}
0=\left[\frac{\partial U}{\partial x}\right] \Delta x+\left[\frac{\partial U}{\partial t}\right] \Delta t, \\
0=\left[\frac{\partial H}{\partial x}\right] \Delta x+\left[\frac{\partial H}{\partial t}\right] \Delta t,
\end{gathered}
$$

since $U$ and $H$ are continuous. We denote the velocity of propagation of the discontinuity, $\Delta x / \Lambda t$, by $\Omega$. Thus the last two equations become

$$
\begin{aligned}
& \Omega\left[\frac{\partial U}{\partial x}\right]+\left[\frac{\partial U}{\partial t}\right]=0, \\
& \Omega\left[\frac{\partial H}{\partial x}\right]+\left[\frac{\partial H}{\partial t}\right]=0,
\end{aligned}
$$


We now have a system of four homogeneous linear equations, eq $76,77,82,83$, in the four unknown discontinuities. Since the discontinuities do not vanish, the determinant of the coefficients must vanish, and this relation gives

$$
\Omega-U= \pm \sqrt{g H} \text {. }
$$

The velocity at the front of a descending wave, as shown in figure 9 , will now be considered. In this case, $\Omega$ is positive, and we see from eq 83 that $[\partial H / \partial x]$ and $[\partial H / \partial t]$ have opposite signs, and since in front of the wave, $\partial H_{1} / \partial t=0, \partial H_{1} / \partial x=0$, then behind the front of the wave, $\partial H_{2} / \partial t>0, \partial H_{2} / \partial x<0$. Now, $U=U_{0}$ and $H=H_{0}$ at the discontinuity, and the velocity of the wave front is

$$
\Omega=U_{0}+\sqrt{g H_{0}}
$$

the positive sign is selected, since $\Omega>0$, regardless of the magnitude of $U_{0}$ and $H_{0}$. We thus see from eq.62 that at the front of such a wave $\Omega=\omega_{0}$. This analysis fails to give any information concerning the change in the magnitude of the discontinuity. Such information may be obtained by using Boussinesq's equation for the velocity of propagation of a wave-volume element. A typical case is discussed in section IV-6, page 498.

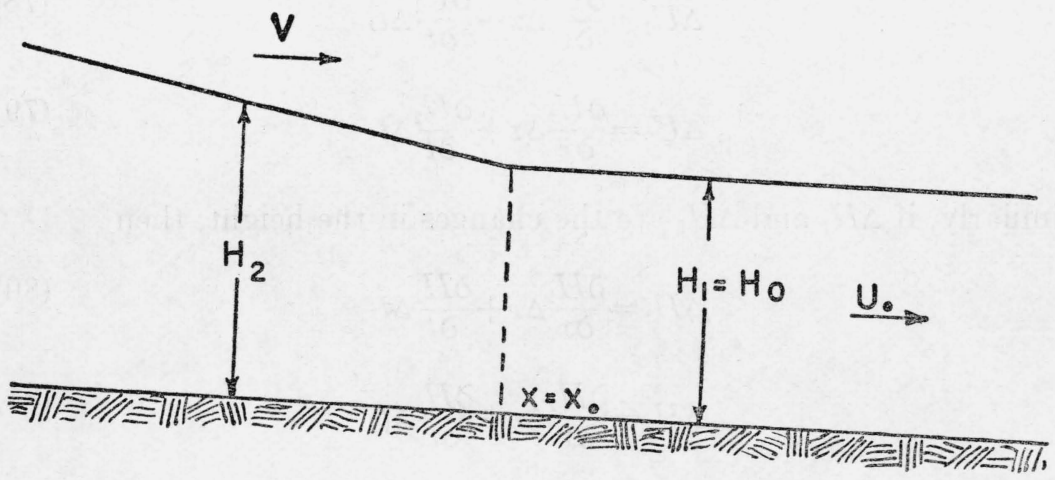

Figure 9.-The front of a descending wave.

\section{EFFECT OF VELOCITY DISTRIBUTION ON THE CELERITY OF SHOR'T WAVES}

In the derivation of the celerity of short waves, given in section III-3, page 478 , it was assumed that $1-\alpha$ is a small fraction that may be neglected. This is generally true in practice, but we now drop this restriction and thereby consider the effect of the velocity distribution on the celerity of short waves. Retaining $\alpha$ and using the assumptions of section III-3, eq 55, 56,69, the equation of motion, eq 53 now becomes

$$
g \frac{\partial h}{\partial x}+\frac{\partial u}{\partial t}+\alpha U_{0} \frac{\partial u}{\partial x}+(1-\alpha) \frac{U_{0}+u}{H_{0}} \frac{\partial h}{\partial t}=0 .
$$

Even though $1-\alpha$ is not negligible, it is still true that in practice 
$\alpha$ is of the same order of magnitude as unity. From eq 69 we have therefore

$$
(1-\alpha) \frac{\omega_{0}-U_{0}}{H_{0}} \frac{\partial h}{\partial t} \frac{h}{H_{0}} \ll \frac{\omega_{0}-U_{0}}{H_{0}} \frac{\partial h}{\partial t} .
$$

Using the relation between $h$ and $u$ obtained in the previous analysis, eq 64 , this inequality reduces to

$$
(1-\alpha) \frac{u}{H_{0}} \frac{\partial h}{\partial t} \ll \frac{\partial u}{\partial t},
$$

and eq 86 thus becomes

$$
g \frac{\partial h}{\partial x}+\frac{\partial u}{\partial t}+\alpha U_{0} \frac{\partial u}{\partial x}+(1-\alpha) \frac{U_{0}}{H_{0}} \frac{\partial h}{\partial t}=0 .
$$

Differentiating with respect to $x$, assuming that $\alpha$ does not vary with $x$, and eliminating the terms containing $u$ by means of eq 59 , we find

$$
\frac{\partial^{2} h}{\partial t^{2}}+2 \alpha U_{0} \frac{\partial^{2} h}{\partial x \partial t}+\left(\alpha U_{0}^{2}-g H_{0}\right) \frac{\partial^{2} h}{\partial x^{2}}=0 .
$$

A particular solution of this is, as before,

$$
h=F\left(x-\omega_{0} t\right),
$$

where $F$ is an arbitrary function, provided $\omega_{0}$ is one of the roots of

$$
\omega_{0}^{2}-2 \alpha U_{0} \omega_{0}+\left(\alpha U_{0}^{2}-g H_{0}\right)=0 .
$$

The two roots are

$$
\omega_{0}=(1+\eta) U_{0} \pm \sqrt{g H_{0}+\left(\eta+\eta^{2}\right) U_{0}^{2}},
$$

where $\eta=\alpha-1$.

This is the formula giving the celerity of short waves when the effect of the velocity distribution is considered. It will be seen that $\eta$ is the excess over unity of the ratio of the mean of the velocities squared, taken over the different points of a section, to the square of the mean velocity. A corresponding expression is given by Boussinesq [3, p. 285, eq 265], which differs from the above only in the values of the coefficients.

It is preferable that the value of $\eta$ for a given case be ascertained by observation. If this is not feasible, a value may be obtained by considering the relation which exists between $\eta$ and $U_{0}$ and $u_{m}$, the latter being the maximum velocity occurring at the surface of a wide channel. A relation of this kind can be derived if it is assumed that in a channel of great width the "velocity defect," that is, the difference between the maximum velocity $u_{m}$ and the velocity $u$ at a point whose height above the bottom is $z$, depends only on the so-called "shear velocity" [5, p. 709] and the relative depth of the water, and is independent of the character of the asperities on the channel bottom. Mathematically speaking,

$$
u_{m}-u=u_{*} f(\zeta),
$$

where $\zeta=z / H_{0}$, and $u_{*}$ is the shear velocity,

$$
u_{*}=\sqrt{i g H_{0}} \text {. }
$$


Multiplying both sides of eq 89 by $d \zeta$, integrating from $z=0$ to $z=H_{0}$ that is, from 0 to 1 , putting

$$
\int_{0}^{1} f(\zeta) d \zeta=N_{1}
$$

and recalling that the mean value of $u$ is $U_{0}$, we obtain

$$
u_{m}-U_{0}=N_{1} u_{*} .
$$

Squaring both sides of eq 89 , we obtain in a similar manner, using eq $88,31,26$,

where

$$
u_{m}^{2}-2 u_{m} U_{0}+(1+\eta) U_{0}^{2}=N_{2} u_{*}^{2},
$$

$\int_{0}^{1} f(\zeta)^{2} d \zeta=N_{2}$. Eliminating the shear velocity, $u_{*}$, between eq 91 and 92 , and solving for $\eta$, we obtain

$$
\eta=\left(\frac{N_{2}}{N_{1}^{2}}-1\right)\left(\frac{u_{m}}{U_{0}}-1\right)^{2},
$$

which is the desired relation expressing $\eta$ in terms of $u_{m}$ and $U_{0}$.

To determine the numerical values of $N_{1}$ and $N_{2}$ we make use of some of Nikuradse's experimental results concerning the velocity distribution in sand-coated circular pipes [14, p. 18-19]. The data in table 1 were obtained by selecting three determinations of the velocity distribution for each of six relative roughnesses. In Nikuradse's paper the data for each relative roughness are given in a separate table, and the three determinations on the extreme right of each table were selected, except in Zahlentafel (table) 13, where the first one and the last two were chosen. In each case, velocity-defect ratios were computed for each value of $y / r$, which corresponds to $z / H_{0}$ in our notation, and the mean of these 18 determinations was entered in table 1. Plotting curves for $f(\zeta)$ and $[f(\zeta)]^{2}$ from these data and integrating numerically, it is found that in circular pipes, $N_{1}=2.52$ and $N_{2}=12.29$. Since the "velocity defect" relation is the same both for circular pipes and for open channels of great width these values of $N_{1}$ and $N_{2}$ may be substituted in eq 93 , which becomes

$$
\eta=0.94\left(\frac{u_{m}}{U_{0}}-1\right)^{2}
$$

An expression for $\eta$ in terms of $\lambda$ may also be derived. Eliminating $u_{m}$ between eq 91 and 92 , we have

$$
\eta=\left(N_{2}-N_{1}^{2}\right) \frac{u_{*}{ }^{2}}{U_{0}^{2}} .
$$

Eliminating $u_{*}$ and $U_{0}$ via eq 90 and 48 :

$$
\eta=\left(N_{2}-N_{1}{ }^{2}\right) \frac{\lambda}{2},
$$


which becomes, when we replace $N_{2}$ and $N_{1}$ by their numerical values

$$
\eta=2.97 \lambda \text {. }
$$

TABLE 1.-Velocity defect as a function of distance from channel bottom.

\begin{tabular}{|c|r||r|c|}
\hline$\frac{z}{H_{0}}$ & $\frac{u_{m}-u}{u_{*}}$ & $\frac{z}{H_{0}}$ & $\frac{u_{m}-u}{u_{*}}$ \\
\cline { 1 - 2 } 0.00 & 12.78 & 0.50 & 1.75 \\
.02 & 9.52 & .60 & 1.23 \\
.04 & 8.09 & .70 & 0.81 \\
.07 & 6.84 & .80 & .45 \\
.10 & 5.99 & .90 & .189 \\
.20 & 5.03 & .96 & .067 \\
.30 & 4.29 & .98 & .030 \\
.40 & 3.21 & 1.00 & .000 \\
\hline
\end{tabular}

It will be recalled that in deriving eq 62 of section I-3 it was assumed that $\alpha$ is nearly unity, that is, that $\eta$ is small, and that this restriction was removed in deriving eq 87. By comparing these two equations we can establish an approximate relation which will show how small $\eta$ must be in order that eq 62 may be used. $A$.

Equation 87 may be approximated by

$$
\omega_{0}=(1+\eta) U_{0} \pm \sqrt{g H_{0}}\left(1+\frac{\eta+\eta^{2}}{2} \frac{U_{0}^{2}}{g H_{0}}\right),
$$

and this reduces to eq 62 if

$$
\eta U_{0} \pm \frac{\eta+\eta^{2}}{2} \frac{U_{0}^{2}}{\sqrt{g H_{0}}} \ll U_{0} \pm \sqrt{g H_{0}}
$$

Dividing by $U_{0}$, neglecting $\eta^{2}$ with respect to $\eta$, using eq 48 , this becomes

$$
\eta\left[1+\frac{1}{2} \sqrt{\frac{2 i}{\lambda}}\right] \ll 1+\sqrt{\frac{\lambda}{2 i}}
$$

or, replacing $\eta$ by its value from eq 95 ,

$$
2.97 \lambda\left[1+\frac{1}{2} \sqrt{\frac{2 i}{\lambda}}\right] \ll 1+\sqrt{\frac{\lambda}{2 i}} .
$$

Bazin's inexhaustible store of experimental data furnishes an example [15] of the improvement obtained by using eq 87 instead of eq 62 . The observed values, together with the results of the two formulas, eq 62 and eq 87 are shown in table 2. These experiments were conducted in the discharge channel of the Grosbois reservoir. This channel was constructed of masonry, with the steep slopes of 0.101 in the upper part and 0.037 in the lower part. Before entering the channel, the water drawn from the reservoir flowed over an apron $11.20 \mathrm{~m}$ wide just below the gates, and which narrowed down to 1.80 $m$ (the width of the channel). The axis of the apron described an are of $90^{\circ}$, with a radius of $40.50 \mathrm{~m}$, in order to join with the channel. As a result of the narrowing of the cross section and the change of direction of the apron, the flowing water was subjected to various reflections at the walls and these complex movements set up waves which passed rapidly down the channel at fairly regular intervals of about 2 
sec (31 per minute). These waves were rather small in height, but their transit was easily observed owing to the slight noise which accompanied them.

1 The observed values of $\omega_{0}$ given in the table 2 are the averages of six individual observations, the \pm term representing the standard deviation. The values of $\eta$ are computed from eq 94. The table shows that the values of $\omega_{0}$ computed by the simple formula, eq 62, are considerably in error, whereas the values of $\omega_{0}$ obtained from eq 87 compare reasonably well with the experimental values.

F. TABLE 2.-Experimental and calculated values of $\omega_{0}$ for torrential flow

\begin{tabular}{|c|c|c|c|c|}
\hline \multicolumn{5}{|c|}{ Experimental data $\left(g=9.81 \mathrm{~m} / \mathrm{sec}^{2}\right)$} \\
\hline$i$ & $H_{0}$ & $U_{0}$ & $u_{m}$ & $\omega_{0}$ \\
\hline $\begin{array}{r}0.101 \\
.037 \\
.037\end{array}$ & $\begin{array}{c}m \\
0.110 \\
.150 \\
.235\end{array}$ & $\begin{array}{l}m / s e c \\
3.785 \\
2.744 \\
3.481\end{array}$ & $\begin{array}{l}m / s e c \\
5.51 \\
3.49 \\
4.55\end{array}$ & $\begin{array}{c}\mathrm{m} / \mathrm{sec} \\
6.25 \pm .07 \\
4.32 \pm .08 \\
5.75 \pm .06\end{array}$ \\
\hline \multicolumn{2}{|c|}{ Computed values eq 62} & \multicolumn{3}{|c|}{ Computed values eq 87} \\
\hline$\omega_{0}$ & Error & $\eta$ & $\omega_{0}$ & Error \\
\hline $\begin{array}{l}m / s e c \\
4.82 \\
3.96 \\
5.00\end{array}$ & $\begin{array}{r}\% \\
-22.9 \\
-8.3 \\
-13.0\end{array}$ & $\begin{array}{r}0.195 \\
.070 \\
.089\end{array}$ & $\begin{array}{l}\mathrm{m} / \mathrm{sec} \\
6.63 \\
4.36 \\
5.65\end{array}$ & $\begin{array}{c}\% \\
+6.1 \\
+0.9 \\
-1.7\end{array}$ \\
\hline
\end{tabular}

\section{SECOND-ORDER THEORY OF WAVE PROPAGATION}

\section{EQUATION OF MEAN FLOW WITH APPRECIABLE VERTICAL ACCELERATION}

Assuming that the liquid is flowing in a wide rectangular channel ( $v=0$, and $R_{v x}=0$ ), that the variation with $z$ of the $x$-component, $u$, of the local velocity is negligible, or $u=U(x, t)$ and that the assumptions made in section III-1 regarding the Reynolds stresses and the orientation of the axes still hold, then the Reynolds equations for turbulent flow, eq 10 become

$$
\begin{gathered}
\frac{\partial U}{\partial t}+U \frac{\partial U}{\partial x}=g i-\frac{1}{\rho} \frac{\partial\left(p-R_{x x}\right)}{\partial x}+\frac{1}{\rho} \frac{\partial R_{z x}}{\partial z}, \\
0=\frac{\partial\left(p-R_{x x}\right)}{\partial y}
\end{gathered}
$$

and

$$
\frac{\partial w}{\partial t}+U \frac{\partial w}{\partial x}+w \frac{\partial w}{\partial z}=-\frac{1}{\rho} \frac{\partial\left(p-R_{x x}\right)}{\partial z}-g .
$$

We shall eliminate $p$ from the eq 98 with the aid of eq 99 , and then eliminate $z$ by integrating over the cross section. 
We begin by evaluating $w$ in terms of the other variables. Since $u=U$ and $v=0$, the condition of continuity eq 8 reduces to

$$
\frac{\partial w}{\partial z}=-\frac{\partial U}{\partial x}
$$

Integrating with respect to $z$, we have, since $w=0$ when $z=0$,

$$
w=-z \frac{\partial U}{\partial x}
$$

which, using a condition of continuity of mean flow, derived from eq 21 on the basis of the assumption that $A$ is proportional to $H$,

reduces to

$$
\frac{\partial H}{\partial t}+U \frac{\partial H}{\partial x}+H \frac{\partial U}{\partial x}=0,
$$

$$
w=\frac{z}{H}\left(\frac{\partial H}{\partial t}+U \frac{\partial H}{\partial x}\right) .
$$

Replacing $w$ in eq 99 by its value from eq 101 and neglecting squares and products of the first derivatives of $H$ and $U$ compared with the second derivative, we obtain, since $H$ and $U$ are functions of $x$ and $t$ only,

$$
\frac{\partial\left(p-R_{x x}\right)}{\partial z}=-\rho g-\frac{\rho z U^{2}}{H}\left(\frac{\partial^{2} H}{\partial x^{2}}+\frac{2}{U} \frac{\partial^{2} H}{\partial x \partial t}+\frac{1}{U^{2}} \frac{\partial^{2} H}{\partial t^{2}}\right) .
$$

Multiplying by $d z$ and integrating,

$$
p-R_{x x}=p_{a}+\rho g(H-z)+\rho \frac{U^{2} H}{2}\left(1-\frac{z^{2}}{H^{2}}\right)\left(\frac{\partial^{2} H}{\partial x^{2}}+\frac{2}{U} \frac{\partial^{2} H}{\partial x \partial t}+\frac{1}{U^{2}} \frac{\partial^{2} H}{\partial t^{2}}\right),
$$

since at $z=H, p-R_{x x}=p_{a}$. We now differentiate the above equation with respect to $x$, again neglecting the products of the lower derivatives as compared with the higher derivatives, and the value thus obtained for $\partial\left(p-R_{x x}\right) / \partial x$ is inserted in eq 98 . The resulting equation is multiplied by $d z$ and integrated from $z=0$ to $z=H$. Since $\left(R_{x z}\right)_{z=0}$ equals $\tau_{0}$, the shear on the channel bottom, and $\left(R_{x z}\right)_{z=H}$ is zero, we get

$$
\frac{\partial U}{\partial t}+U \frac{\partial U}{\partial x}+g \frac{\partial H}{\partial x}+\frac{H U^{2}}{3}\left(\frac{\partial^{3} H}{\partial x^{3}}+\frac{2}{U} \frac{\partial^{3} H}{\partial x^{2} \partial t}+\frac{1}{U^{2}} \frac{\partial^{3} H}{\partial x \partial t^{2}}\right)=g i-\frac{\lambda U^{2}}{2 H},
$$

upon replacing $\tau_{0}$ by its value from eq 45 .

This is the equation of mean flow for a wide rectangular channel where the vertical accelerations are not negligible but the horizontal velocities in a section are practically uniform.

\section{HEIGHT OF WAVE AS A FUNCTION OF $x$ AND $t$}

Replacing $\partial U / \partial x$ in eq 102 by its value obtained by solving eq 100 , and multiplying through by $H$, we obtain

$$
\begin{aligned}
H \frac{\partial U}{\partial t}-U \frac{\partial H}{\partial t}+\left(g H-U^{2}\right) \frac{\partial H}{\partial x}+ & \frac{H^{2} U^{2}}{3}\left(\frac{\partial^{3} H}{\partial x^{3}}\right. \\
& \left.+\frac{2 \partial^{3} H}{U \partial x^{2} \partial t}+\frac{1}{U^{2}} \frac{\partial^{3} H}{\partial x \partial t^{2}}\right)=g H i-\frac{\lambda}{2} U^{2} .
\end{aligned}
$$


We again introduce the expressions

and

$$
\left.\begin{array}{l}
H(x, t)=H_{0}+h(x, t), \\
U(x, t)=U_{0}+u(x, t) .
\end{array}\right\}
$$

Substituting in eq 100 , we have

$$
\frac{\partial h}{\partial t}+H_{0} \frac{\partial u}{\partial x}+U_{0} \frac{\partial h}{\partial x}+h \frac{\partial u}{\partial x}+u \frac{\partial h}{\partial x}=0,
$$

while eq 103 becomes

$$
\begin{aligned}
H_{0} \frac{\partial u}{\partial t}-U_{0} \frac{\partial h}{\partial t} & +\left(g H_{0}-U_{0}^{2}\right) \frac{\partial h}{\partial x}+h \frac{\partial u}{\partial t}-u \frac{\partial h}{\partial t}+\left(g h-2 U_{0} u\right) \frac{\partial h}{\partial x} \\
& +\frac{H_{0}^{2} U_{0}^{2}}{3}\left(\frac{\partial^{3} h}{\partial x^{3}}+\frac{2}{U_{0}} \frac{\partial^{3} h}{\partial x^{2} \partial t}+\frac{1}{U_{0}^{2}} \frac{\partial^{3} h}{\partial x \partial t^{2}}\right)=g H i-\frac{\lambda}{2} U^{2}
\end{aligned}
$$

when $u^{2}$ is neglected with respect to $U_{0}^{2}$, and in the term containing the third derivatives, $u$ and $h$ are neglected in comparison with $U$ and $H$. Various assumptions as to the nature of the right-hand member will be made in later sections.

The terms of small order in the left-hand members of the above equations, that is, the last two in eq 104 and the last four in eq 105, are simplified by introducing values from the first approximate solution:

and

$$
u=\frac{\omega_{0}-U_{0}}{H_{0}} h,
$$

$$
\frac{\partial h}{\partial t}=-\omega_{0} \frac{\partial h}{\partial x}
$$

From the latter and eq 61, we obtain the further relation

$$
\frac{\partial^{2} h}{\partial t^{2}}=\omega_{0}^{2} \frac{\partial^{2} h}{\partial x^{2}} \text {. }
$$

For the sake of brevity, we introduce the new variables

and

$$
\left.\begin{array}{l}
k_{1}=1-\frac{3 U_{0}}{\omega_{0}} \\
k_{2}=1-\frac{U_{0}}{\omega_{0}},
\end{array}\right\}
$$

and we also make use of the equation

$$
g H_{0}=\left(\omega_{0}-U_{0}\right)^{2},
$$

which is derived from eq 62 . On the basis of the above relations, then eq 104 and 105 become 


$$
\frac{\partial h}{\partial t}+H_{0} \frac{\partial u}{\partial x}+U_{0} \frac{\partial h}{\partial x}+\left(\omega_{0}-U_{0}\right) \frac{\partial}{\partial x}\left(\frac{h^{2}}{H_{0}}\right)=0 ;
$$

and

$$
\begin{gathered}
H_{0} \frac{\partial u}{\partial t}-U_{0} \frac{\partial h}{\partial t}+\left(g H_{0}-U_{0}^{2}\right) \frac{\partial h}{\partial x}+\omega_{0}\left(\omega_{0}-U_{0}\right) \frac{\partial}{\partial x}\left(\frac{k_{1}}{2} \frac{h^{2}}{H_{0}}\right. \\
\left.+\frac{k_{2} H_{0}^{2}}{3} \frac{\partial^{2} h}{\partial x^{2}}\right)=g H i-\frac{\lambda}{2} U^{2}
\end{gathered}
$$

We now differentiate eq 108 with respect to $t$. In differentiating the last term, which is of the second order, we use the relation

$$
\left(\frac{\partial}{\partial t}\right)=-\omega_{0}\left(\frac{\partial}{\partial x}\right)
$$

since these small terms may be treated as functions of $x-\omega_{0} t[1, p .73]$. We next differentiate eq 109 with respect to $x$, and subtract this equation from the one obtained from eq 108. The result is

$$
\begin{aligned}
\frac{\partial^{2} h}{\partial t^{2}} & +2 U_{0} \frac{\partial^{2} h}{\partial x \partial t}-\left(g H_{0}-U_{0}^{2}\right) \frac{\partial^{2} h}{\partial x^{2}} \\
& -\omega_{0}\left(\omega_{0}-U_{0}\right) \frac{\partial^{2}}{\partial x^{2}}\left(\frac{2+k_{1}}{2}\left(\frac{h^{2}}{H_{0}}\right)+\frac{k_{2} H_{0}^{2}}{3} \frac{\partial^{2} h}{\partial x^{2}}\right)=-\frac{\partial}{\partial x}\left(g H i-\frac{\lambda}{2} U^{2}\right)
\end{aligned}
$$

which is the fundamental equation for determining $h$ as a function of $x$ and $t$.

This equation, which was derived from eq 102, has the same range of validity, namely, it applies to flow in wide rectangular channels where the horizontal velocities in a section are practically uniform. This equation takes account of vertical accelerations, finite wave heights, surface curvature, and friction.

\section{CELERITY OF A WAVE-VOLUME ELEMENT}

In order to integrate eq 111, the concept of celerity of a wave-volume element, an essential simplification due to Boussinesq [3, p. 451], is introduced. To define this concept, we consider the volume of the wave per unit width of channel, above the undisturbed primitive level of the liquid and between $x$, an arbitrary point, and $\infty$, for a descending wave, and between $x$ and $-\infty$, for an ascending wave. Denoting this volume by $\sigma$, we have

$$
\sigma=\int_{x}^{\infty} h d x \text { and } \sigma=\int_{-\infty}^{x} h d x,
$$

for descending and ascending waves, respectively. Recalling the convention of signs introduced in section III-3, the above equations become

$$
\sigma= \pm \int_{x}^{ \pm \infty} h d x
$$

Imagine a plane, normal to the channel axis at the point $x$, and which moves in such a way that the wave volume in front of it, $\sigma$, remains constant. This velocity is the celerity of the volume element $\sigma$, and 
is denoted by $\omega=\omega(x, t)$. Differentiating eq 112 with respect to $t$, we have

$$
0= \pm \int_{x}^{ \pm \infty} \frac{\partial h}{\partial t} d x \mp h \omega
$$

since by definition $d x / d t=\omega, \sigma$ remains constant, and we assume that the surface of the liquid is undisturbed at infinity, that is, $h$ vanishes at $\pm \infty$. Differentiating the above equation with respect to $x$, we have, regardless of choice of sign,

$$
\frac{\partial h}{\partial t}+\frac{\partial}{\partial x}(h \omega)=0,
$$

which gives the relation obtaining between $\omega$ and $h$.

We now introduce the value of $\partial h / \partial t$ from eq 113 into the first two terms of eq 111, thus obtaining

$$
\begin{aligned}
-\frac{\partial^{2}}{\partial x \partial t}(h \omega) & -2 U_{0} \frac{\partial^{2}}{\partial x^{2}}(h \omega)-\left(g H_{0}-U_{0}^{2}\right) \frac{\partial^{2} h}{\partial x^{2}} \\
& -\omega_{0}\left(\omega_{0}-U_{0}\right) \frac{\partial^{2}}{\partial x^{2}}\left(\frac{2+k_{1}}{2} \frac{h^{2}}{H_{0}}+\frac{k^{2} H_{0}^{2}}{3} \frac{\partial^{2} h}{\partial x^{2}}\right)=-\frac{\partial}{\partial x}\left(g H i-\frac{\lambda}{2} U^{2}\right) .
\end{aligned}
$$

This equation is immediately integrable once with respect to $x$. The arbitrary function of integration vanishes as a result of the previous assumption that the surface of the liquid is undisturbed at infinity. We thus have

$$
\begin{aligned}
\frac{\partial}{\partial t}(h \omega)+2 U_{0} & \frac{\partial}{\partial x}(h \omega)+\left(g H_{0}-U_{0}^{2}\right) \frac{\partial h}{\partial x} \\
& +\omega_{0}\left(\omega_{0}-U_{0}\right) \frac{\partial}{\partial x}\left(\frac{2+k_{1}}{2} \frac{h^{2}}{H_{0}}+\frac{k_{2} H_{0}^{2}}{3} \frac{\partial^{2} h}{\partial x^{2}}\right)=g H i-\frac{\lambda}{2} U^{2} .
\end{aligned}
$$

We next transform eq 114 into a form which can be integrated. In this process it is necessary to operate only on the first three terms in the left-hand member. If we assume that $\omega / \omega_{0}$ is near to unity, we may write as an approximation

$$
\omega=\omega_{0}(1+\delta(x, t)) .
$$

Substituting this value of $\omega$ in the terms under consideration, we obtain

$$
\omega_{0} \frac{\partial}{\partial t}(h+h \delta)+2 \omega_{0} U_{0} \frac{\partial}{\partial x}(h+h \delta)+\left(g H_{0}-U_{0}^{2}\right) \frac{\partial h}{\partial x} .
$$

Now using eq 113 and 107 we obtain

$$
-\omega_{0} \frac{\partial}{\partial x}(h \omega)+\omega_{0} \frac{\partial}{\partial t}(h \delta)+2 \omega_{0} U_{0} \frac{\partial}{\partial x}(h+h \delta)+\left(\omega_{0}{ }^{2}-2 \omega_{0} U_{0}\right) \frac{\partial h}{\partial x} .
$$

Applying eq 115 again to the first term and eq 110 to the second term (which can be done because $h \delta$ is a small quantity), and cancelling terms, we obtain

$$
-2 \omega_{0}{ }^{2} \frac{\partial}{\partial x}(h \delta)+2 \omega_{0} U_{0} \frac{\partial}{\partial x}(h \delta),
$$


which can be written

$$
-\omega_{0}\left(\omega_{0}-U_{0}\right) \frac{\partial}{\partial x}(2 h \delta)
$$

Hence eq 114 transforms into

$$
\begin{aligned}
-\omega_{0}\left(\omega_{0}-U_{0}\right) \frac{\partial}{\partial x}(2 h \delta)+\omega_{0}\left(\omega_{0}-U_{0}\right) & \frac{\partial}{\partial x} \\
& \left(\frac{2+k_{1}}{2}\left(\frac{h^{2}}{H_{0}}\right)+\frac{k_{2} H_{0}^{2}}{3} \frac{\partial^{2} h}{\partial x^{2}}\right)=g H i-\frac{\lambda}{2} U^{2},
\end{aligned}
$$

which, when integrated, gives

$$
\delta=\frac{2+k_{1}}{4} \frac{h}{H_{0}}+\frac{k_{2} H_{0}^{2}}{6 h} \frac{\partial^{2} h}{\partial x^{2}}-\frac{1}{2 h \omega_{0}\left(\omega_{0}-U_{0}\right)} \int_{ \pm \infty}^{x}\left(g H i-\frac{\lambda}{2} U^{2}\right) d x,
$$

and hence from eq 115

$$
\frac{\omega}{\omega_{0}}=1+\frac{2+k_{1}}{4} \frac{h}{H_{0}}+\frac{k_{2} H_{0}^{2}}{6 h} \frac{\partial^{2} h}{\partial x^{2}}+\frac{1}{2 h \omega_{0}\left(\omega_{0}-U_{0}\right)} \int_{x}^{ \pm \infty}\left(g H i-\frac{\lambda}{2} U^{2}\right) d x .
$$

This is a general expression for determining the effects of wave height, curvature of the wave profile, and friction, on the celerity of a. wave-volume element. The integral containing the friction term. requires further consideration before the actual wave profile can be determined as a function of time.

It remains to evaluate that part $u$ of the velocity $U$ under the wave in eq 52. Transforming the equation of continuity, eq 100, by means of eq 52 ,

$$
\frac{\partial h}{\partial t}+\frac{\partial}{\partial x}\left(U_{0}+u\right)\left(H_{0}+h\right)=0 .
$$

The result of subtracting eq 113 from the above is

$$
\frac{\partial}{\partial x}\left[\left(U_{0}+u\right)\left(H_{0}+h\right)-h \omega\right]=0 .
$$

Integrating, and using the boundary condition at $x=\infty$, for all times $t, h=0$, and $u=0$, we have

$$
\left(U_{0}+u\right)\left(H_{0}+h\right)-h \omega=U_{0} H_{0},
$$

or

$$
u=\frac{\omega-U_{0}}{H_{0}+h} h
$$

$$
\begin{aligned}
\text { 4. DEFORMATION OF } & \text { THE WAVE PROFILE, ASSUMING } \\
& g H i-\frac{\lambda}{2} U^{2}=0
\end{aligned}
$$

A natural assumption, in proceeding from eq 116, is that the righthand member $\left(g H i-\lambda U^{2} / 2\right)$ is negligible. From eq 56 this implies that 
the waves are short, as described in section III-3. On this basis, eq 116 becomes

$$
\omega=\omega_{0}\left(1+\frac{2+k_{1}}{4} \frac{h}{H_{0}}+\frac{k_{2} H_{0}^{2}}{6 h} \frac{\partial^{2} h}{\partial x^{2}}\right),
$$

or when we introduce the values of $k_{1}, k_{2}$, and $\omega_{0}$ from eq 106 and 62 ,

$$
\omega-U_{0}= \pm \sqrt{g H_{0}}\left(1+\frac{3 h}{4 H_{0}}+\frac{H_{0}^{2}}{6 h} \frac{\partial^{2} h}{\partial x^{2}}\right) .
$$

Comparison with an earlier paper [1, eq 112] shows that this expression is equivalent to that for the propagation of an irrotational wave relative to the mean velocity of flow, $U_{0}$. The assumption that $g H i-\lambda U^{2} / 2$ is equal to zero is thus tantamount to assuming that the only effect of friction and slope is to superpose a constant velocity $U_{0}$ on the wave, which itself is propagated without any change of shape due to frictional damping or to the $x$-component of gravity.

The deformation of the wave profile, in a short time interval, is determined by using eq 119 . Equation 113 can be written

$$
\frac{\partial h}{\partial t}+\omega_{0} \frac{\partial h}{\partial x}+\frac{\partial}{\partial x} h\left(\omega-\omega_{0}\right)=0 .
$$

For small values of $t$, the integral of the above equation is

$$
h=f\left(x-\omega_{0} t\right)-t \frac{\partial}{\partial x}\left[h\left(\omega-\omega_{0}\right)\right] .
$$

In verifying this solution, we must use eq 110 when differentiating the second term on the right, which necessitates assuming that this term is small compared with the first term. In other words, the magnitude of the time interval in which this approximation is permissible varies inversely with $\partial\left[h\left(\omega-\omega_{0}\right)\right] / \partial x$. Clearly, since at $t=0$, we have $h=f(x)$, the function $f$ represents the wave profile at the fiducial instant. Finally, using eq 119 and 62, eq 120 can be thrown into the form

$$
h=f\left(x-\omega_{0} t\right) \mp t \sqrt{g H_{0}} \frac{\partial}{\partial x}\left(\frac{3 h^{2}}{4 H_{0}}+\frac{H_{0}^{2}}{6} \frac{\partial^{2} h}{\partial x^{2}}\right) .
$$

For a discussion of the deformation of a wave of negligible curvature, propagated without damping, the reader is referred to the earlier paper $[1$, p. 89]. All the formulas on the page cited are directly applicable, provided the expression $\sqrt{g H}(1+\ldots)$ is replaced by $U_{0} \pm \sqrt{g H_{0}}(1+\ldots)$, wherever it occurs. This, again, is merely equivalent to superposing the velocity of flow $U_{0}$, on the velocity of propagation of the wave-volume element.

\section{EFFECT OF FRICTION AND SLOPE OF BED ON THE PROPAGATION OF WAVES}

In the above solution of eq 116 , the term $g H i-\lambda U^{2} / 2$ was ignored. If the wave is short, the influence of this term is negligible, as explained in section III-3. For long waves, however, these effects become 
appreciable at distances sufficiently removed from the head of the wave. The problem of determining these effects is considered separately for the two cases, (I) $i>0$ and $U_{0}>0$, and (II) $i=0$ and $U_{0}=0$. The first case will now be considered.

From eq 52, we have, after neglecting $u^{2}$ in comparison with $U_{0}^{2}$,

$$
g H i-\frac{\lambda}{2} U^{2}=g H_{0} i-\frac{\lambda}{2} U_{0}^{2}+g h i-\lambda U_{0} u .
$$

Considering the first two terms of the Taylor expansion of $\lambda=\lambda(H)$ at $H_{0}$,

$$
\lambda=\lambda_{0}+\left(\frac{d \lambda}{d H}\right) h,
$$

where $\lambda_{0}=\lambda\left(H_{0}\right)$, and $d \lambda / d H$ is evaluated at $H=H_{0}$. Replacing $\lambda$ by the above value, and neglecting the small $h u$ term, eq 122 becomes

$$
g H i-\frac{\lambda}{2} U^{2}=g H_{0} i-\frac{\lambda_{0}}{2} U_{0}^{2}+g h i-\frac{1}{2} \frac{d \lambda}{d H} h U_{0}^{2}-\lambda_{0} U_{0} u .
$$

From eq 48 we have

$$
g H_{0} i-\frac{\lambda_{0}}{2} U_{0}^{2}=0
$$

that is, in uniform flow the effects of slope and friction are in equilibrium, and thus

$$
g H i-\frac{\lambda}{2} U^{2}=g h i-\frac{1}{2} \frac{d \lambda}{d H} h U_{0}^{2}-\lambda_{0} U_{0} u .
$$

Evaluating $d \lambda / d H$ by means of eq 51 and 124 , and introducing the value of $u$ from the first approximation, eq 64 , the above equation becomes

where

$$
g H i-\frac{\lambda}{2} U^{2}=-2\left(\omega_{0}-U_{0}\right) h f_{1},
$$

$$
f_{1}=\frac{3 \omega_{0}-5 U_{0}}{3 U_{0}\left(\omega_{0}-U_{0}\right)} g i \text {. }
$$

Since from eq $112, d \sigma / d x=\mp h$, eq 125 can be written

$$
g H i-\frac{\lambda}{2} U^{2}= \pm 2 \omega_{0}\left(\omega_{0}-U_{0}\right) \frac{d}{d x}\left(\frac{\sigma f_{1}}{\omega_{0}}\right) .
$$

Substituting this in eq 116 , we have

$$
\omega=\omega_{0}\left(1+\frac{2+k_{1}}{4} \frac{h}{H_{0}}+\frac{k_{2} H_{0}^{2}}{6 h} \frac{\partial^{2} h}{\partial x^{2}} \mp \frac{\sigma f_{1}}{\omega_{0} h}\right),
$$

or introducing the values of $k_{1}$ and $k_{2}$ from eq 106 , and using eq 62

$$
\omega-U_{0}= \pm\left(\sqrt{g H_{0}}\left(1+\frac{3 h}{4 H_{0}}+\frac{H_{0}^{2}}{6 h} \frac{\partial^{2} h}{\partial x^{2}}\right)-\frac{\sigma f_{1}}{h}\right) .
$$


This integral of eq 116 is due to Boussinesq [3, p. 451], except that the value of $f_{1}$ given above is new, being based on Manning's quadratic law of resistance developed in section III-2.

We now consider the sign of the quantity $\sigma f_{1} / h$. If the intumescence is everywhere positive or everywhere negative; that is, for all $x, h \geqq 0$ or $h \leqq 0$, then $\sigma$ has the same sign as $h$, and $\sigma / h$ is positive. At the head of the wave this ratio vanishes, since $\sigma$ is of higher order than $h$, and it increases toward the rear of the wave. Since $f_{1}$ is constant for a given channel and depth of water in uniform flow, the absolute value of the term varies in the manner just described, and its sign is the same as that of $f_{1}$. Upon examining eq 126 we find that $f_{1}$ is positive if (1) $U_{0}>\omega_{0}$ or (2) $U_{0}<\omega_{0}$ and $3 \omega_{0}>5 U_{0}$, and negative if $(3) U_{0}<\omega_{0}$ and $3 \omega_{0}<5 U_{0}$. Comparison with eq 62 shows that (1) applies to all descending waves, (2) to descending waves where $U_{0}^{2}<(9 / 4) g H_{0}$, (3) to descending waves where $U_{0}^{2}>(9 / 4) g H_{0}$. Thus, except in the last case, the combined effect of friction and slope on the damping of the wave is to cause a diminution of the absolute value of the velocity of propagation with respect to the velocity of flow of the various parts of the intumescence, the effect increasing with increasing distance from the head of the wave. The last case is of special interest. It has been shown elsewhere [16] that if $U_{0}^{2}>(9 / 4) g H_{0}$, the waves become unstable, a phenomenon which is apparently closely related to the origin of roll waves.

\section{DEFORMATION OF A STRAIGHT SLOPING FRONT}

We suppose that the wave is descending (fig. 10). Denoting the slope of the front by $\beta, \beta=-\tan \varphi$. Since we are only considering waves whose profiles lie above the free surface, $\beta$ is always positive. Denoting, at $t=0$, the position of the front of the wave by $x_{0}$, and the value of the slope by $\beta_{0}$, the equation of the wave profile is thus,

$$
\begin{array}{ll}
h=\beta_{0}\left(x_{0}-x\right), & x \leqq x_{0}, t=0 ; \\
h=0, & x \geqq x_{0}, t=0 .
\end{array}
$$

Equation 112 then becomes

$$
\left.\begin{array}{ll}
\sigma=\frac{\beta_{0}\left(x_{0}-x\right)^{2}}{2}, & x \leqq x_{0}, t=0 \\
\sigma=0 & x \geqq x_{0}, t=0
\end{array}\right\}
$$

Introducing the equation for the velocity of propagation of a wave volume element, eq 128, we obtain from the above equations

$$
\omega=\omega_{0}\left[1+\left(\frac{2+k_{1}}{4 H_{0}} \beta_{0}-\frac{f_{1}}{2 \omega_{0}}\right)\left(x_{0}-x\right)\right], x \leqq x_{0}, t=0 .
$$

If we write

$$
\theta_{0}=\frac{2+k_{1}}{4 H_{0}} \omega_{0} \beta_{0}-\frac{f_{1}}{2}
$$

then the last equation becomes

$$
\omega-\omega_{0}=\theta_{0}\left(x_{0}-x\right), \quad x \leqq x_{0}, t=0 .
$$




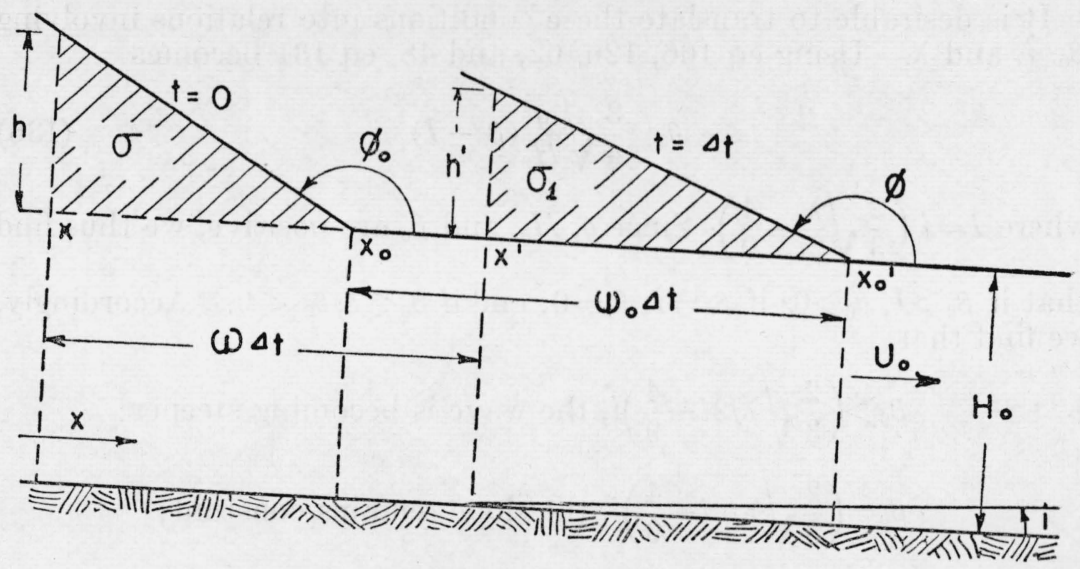

Figure 10.-Deformation of the linear front of a descending wave.

Now let the position of the front of the wave at $t=\Delta t$ be $x_{0}{ }^{\prime}$ (fig. 10). The section which at time $t=0$ had the volume $\sigma$ in front of it has moved from $x$ to $x^{\prime}$, and the height has changed from $h$ to $h^{\prime}$. Since the wave front moves with the velocity $\omega_{0}$ (section III-4), while the section at $x$ moves with velocity $\omega$, we have

$$
x_{0}^{\prime}-x^{\prime}=\left(\omega_{0}-\omega\right) \Delta t+\left(x_{0}-x\right), \quad x<x_{0}, t=0 .
$$

Using eq 132 , we obtain

$$
x_{0}{ }^{\prime}-x^{\prime}=\left(1-\theta_{0} \Delta t\right)\left(x_{0}-x\right), \quad x<x_{0}, t=0 .
$$

But the volume $\sigma$ in front of $x$ is equal to the volume $\sigma_{1}$ in front of $x^{\prime}$, and thus we have from eq 130 and 133

$$
\sigma_{1}=\frac{\beta_{0}}{2}\left(x_{0}^{\prime}-x^{\prime}\right)^{2}\left(1+2 \theta_{0} \Delta t\right) .
$$

Comparison with eq 130 shows that during the time $\Delta t$ the linear character of the wave front is preserved, but the slope has changed from $\beta_{0}$ to $\beta^{\prime}$,

$$
\beta^{\prime}=\left(1+2 \theta_{0} \Delta t\right) \beta_{0}
$$

Putting

$$
\beta^{\prime}=\beta_{0}+\frac{d \beta}{d t} \Delta t
$$

we have from eq 134

$$
\frac{d \beta}{d t}=2 \theta_{0} \beta_{0}, \quad t=0
$$

which enables us to determine the variation of the slope. Since $\beta_{0}$ is positive, we find that if $\theta_{0}>0$, the wave is becoming steeper, if $\theta_{0}=0$, the slope remains constant, if $\theta_{0}<0$, the wave is flattening out. 
It is desirable to translate these conditions into relations involving $\beta_{0}$. $i$, and $\lambda$. Using eq $106,126,62$, and 48 , eq 131 becomes

$$
\theta_{0}=\frac{3}{4} \sqrt{\frac{g}{H_{0}}}\left(\beta_{0}-I\right)
$$

where $I=i\left(\frac{2}{3} \sqrt{\frac{\lambda}{2 i}}-\frac{4}{9}\right)$. Since $g, H_{0}$, and $\beta_{0}$ are positive, we thus find that if $\beta_{0}>I, \theta_{0}>0$; if $\beta_{0}=I, \theta_{0}=0$; and if $\beta_{0}<I, \theta_{0}<0$. Accordingly, we find that,

$$
\begin{aligned}
& \beta_{0}>\left(\frac{2}{3} \sqrt{\lambda / 2 i}-\frac{4}{9}\right) i, \text { the wave is becoming steeper; } \\
& \beta_{0}=\left(\frac{2}{3} \sqrt{\lambda / 2 i}-\frac{4}{9}\right) i, \text { the slope remains constant; } \\
& \beta_{0}<\left(\frac{2}{3} \sqrt{\lambda / 2 i}-\frac{4}{9}\right) i, \text { the wave is flattening out. }
\end{aligned}
$$

We now derive an expression for $\beta$ as function of time. Since it has been demonstrated that the wave front conserves its linear character, eq 135 holds for any value of $t$, and we obtain, using eq 135 and 136 and discarding the subscript which refers to $t=0$,

$$
\frac{d \beta}{d t}=\frac{3}{2} \sqrt{\frac{g}{H_{0}}}\left(\beta^{2}-I \beta\right) .
$$

The solution of this differential equation for the initial condition $\beta=\beta_{0}$ at $t=0$ is

$$
\beta=\frac{\beta_{0} I}{\beta_{0}-\left(\beta_{0}-I\right) \exp \frac{3}{2} \sqrt{\frac{g}{H_{0}}} I t} .
$$

If $I=0$, the solution of eq 137 becomes

$$
\beta=\frac{\beta_{0}}{1-\frac{3}{2} \sqrt{\frac{g}{H_{0}}} \beta_{0} t},
$$

and the slope continually increases.

We next suppose that the wave is ascending (fig. 11). Denoting the slope of the front by $\beta, \beta=\tan \varphi$. We again suppose that the wave profile lies above the free surface, which makes $\beta$ positive. Using the same method of reasoning as in the above, we obtain corresponding to eq 135 and 136 ,

where

$$
\begin{aligned}
\frac{d \beta}{d t} & =2 \theta_{0} \beta_{0}, \\
\theta_{0} & =\frac{3}{4} \sqrt{\frac{g}{H_{0}}}\left(-J+\beta_{0}\right), \\
J & =\left(\frac{2}{3} \sqrt{\frac{\lambda}{2 i}}+\frac{4}{9}\right) i .
\end{aligned}
$$


Thus, if $\beta_{0}>\left(\frac{2}{3} \sqrt{\frac{\lambda}{2 i}}+\frac{4}{9}\right) i$, the wave is becoming steeper;

$\beta_{0}=\left(\frac{2}{3} \sqrt{\frac{\lambda}{2 i}}+\frac{4}{9}\right) i$, the slope remains constant;

$\beta_{0}<\left(\frac{2}{3} \sqrt{\frac{\lambda}{2 i}}+\frac{4}{9}\right) i$, the wave is flattening out.

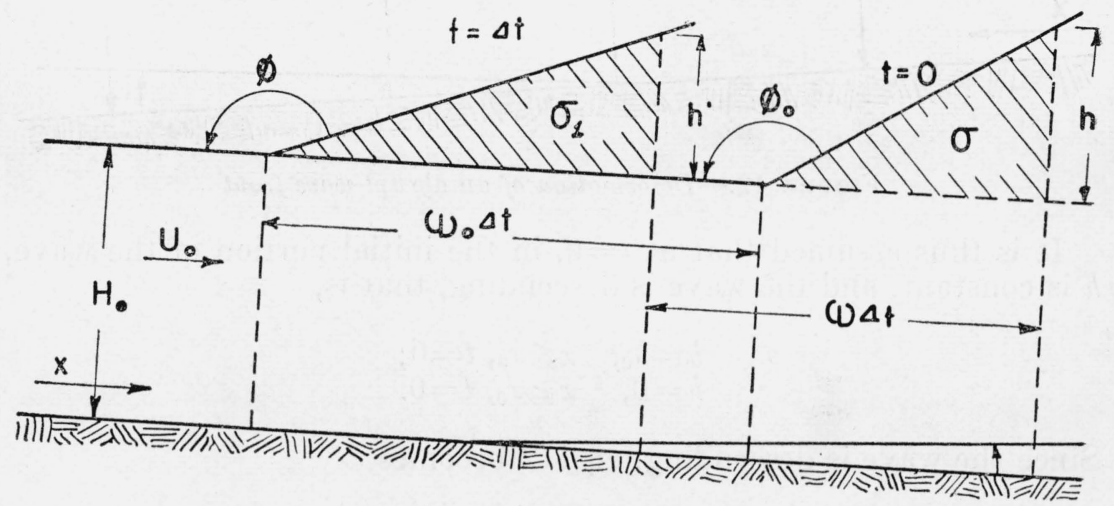

FigURE 11.-Deformation of the linear front of an ascending wave.

The expression for $\beta$ as function of time, becomes

$$
\beta=\frac{\beta_{0} J}{\beta_{0}-\left(\beta_{0}-J\right) \exp \frac{3}{2} \sqrt{\frac{g}{H_{0}}} J t},
$$

and if $J=0$, which for ascending waves can only be the case if the slope, $i$, vanishes, the latter reduces again to

$$
\beta=\frac{\beta_{0}}{1-\frac{3}{2} \sqrt{\frac{g}{H_{0}}} \beta_{0} t},
$$

and the slope of the wave is continually increasing.

If it is desired to express $\beta$ as a function of the distance $x_{0}{ }^{\prime}-x_{0}$ traversed by the wave, this is obtained by introducing $\left(x_{0}^{\prime}-x_{0}\right) / \omega_{0}$ in place of $t$ in the various expressions giving $\beta$ as a function of $t$, since the head of wave is traveling with the velocity $\omega_{0}$.

\section{CHANGE OF HEIGHT OF AN ABRUPT WAVE FRONT}

In a wave having an abrupt wave front there is a discontinuity of height at the wave front (fig. 12). If for some considerable distance behind the front, the height is constant, the change of height with time, or preferably with the displacement of the front, can be readily determined, using the Boussinesq formula for the velocity of propagation of a volume element. 
502 Journal of Research of the National Bureau of Standards

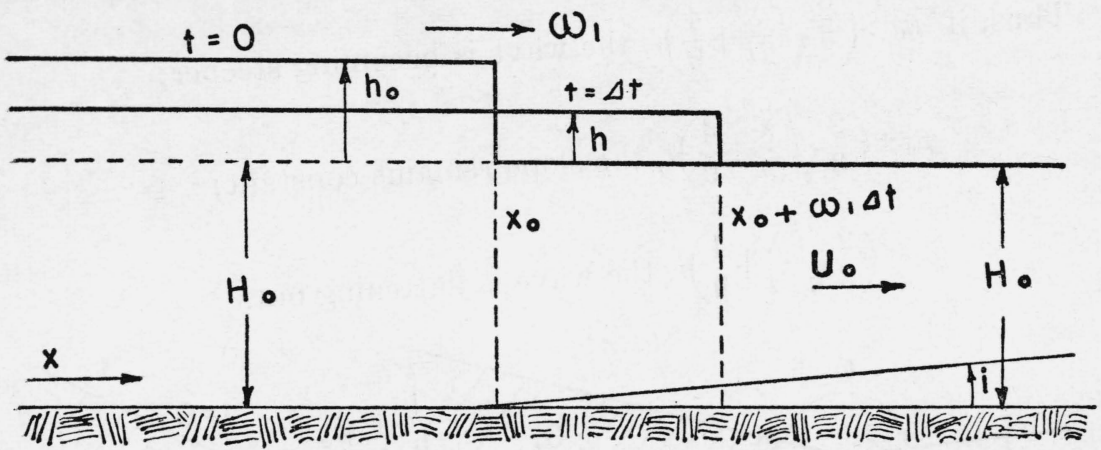

FigURE 12.-Deformation of an abrupt wave front

It is thus assumed that at $t=0$, in the initial portion of the wave, $h$ is constant, and the wave is descending, that is,

$$
\begin{array}{ll}
h=h_{0}, & x \leqq x_{0}, t=0 ; \\
h=0, & x \geqq x_{0}, t=0 .
\end{array}
$$

Since the wave is descending, eq 112 becomes

$$
\begin{array}{ll}
\sigma=h_{0}\left(x_{0}-x\right), & x \leqq x_{0}, t=0 ; \\
\sigma=0, & x \geqq x_{0}, t=0 .
\end{array}
$$

The velocity of propagation of a wave-volume element is thus from eq $128,106,62$

$$
\omega=\omega_{0}\left[1+\frac{3}{4} \frac{\sqrt{g H_{0}}}{\omega_{0}} \frac{h_{0}}{H_{0}}+\frac{\left(x-x_{0}\right) f_{1}}{\omega_{0}}\right], \quad x \leqq x_{0}, t=0 .
$$

From this it follows that the velocity of the wave front, $\omega_{1}$, is

$$
\omega_{1}=\omega_{0}\left[1+\frac{3 \sqrt{g H_{0}}}{4} \frac{h_{0}}{\omega_{0}}\right], \quad t=0 .
$$

Since the height is constant behind the wave front, $\partial h / \partial x=0$, and we have

$$
\frac{d h}{d t}=\frac{\partial h}{\partial t}, \quad x<x_{0}, t=0
$$

and from eq 113

$$
\frac{\partial h}{\partial t}=-h_{0} \frac{\partial \omega}{\partial x}, \quad x<x_{0}, t=0 .
$$

Differentiating eq 142 with respect to $x, \partial \omega / \partial x=f_{1}$, and eq 145 now becomes

$$
\frac{d h}{d t}=-f_{1} h_{0}, \quad x<x_{0}, t=0 .
$$


We consider two distinct values of $x$ at $t=0$, both behind the wave front, denoting them by $x_{1}$ and $x_{2}$, and write $h_{1}=h\left(x_{1}\right), h_{2}=h\left(x_{2}\right)$. After an interval of time $\Delta t$, let the height of the corresponding wave elements be $h_{1}{ }^{\prime}$ and $h_{2}{ }^{\prime}$. Now

$$
h_{1}^{\prime}-h_{1}=\frac{d h}{d t} \Delta t=-f_{1} h_{0} \Delta t
$$

and

$$
h_{2}^{\prime}-h_{2}=\frac{d h}{d t} \Delta t=-f_{1} h_{0} \Delta t .
$$

Since $h_{1}=h_{2}=h_{\theta}$, we see that $h_{1}{ }^{\prime}=h_{2}{ }^{\prime}$. This shows that the constancy of height of the wave front is preserved. Replacing $h_{0}$ by $h$ in eq 143 , we see that the velocity of the wave front $\omega_{1}$ is

$$
\omega_{1}=\omega_{0}\left[1+\frac{3}{4} \frac{\sqrt{g H_{0}}}{\omega_{0}} \frac{h}{H_{0}}\right], t=t .
$$

Again replacing $h_{0}$ by $h$, eq 146 now becomes

$$
\frac{d h}{d t}=-f_{1} h, \quad x<x_{0}+\int_{0}^{t} \omega_{1} d t .
$$

Integrating this equation, and using the initial condition $h=h_{0}$ at $t=0$, we obtain $h$ as a function of $t$

$$
h=h_{0} e^{-f_{1} t}, \quad x<x_{0}+\int_{0}^{t} \omega_{1} d t .
$$

If it is desired to determine $h$ as a function of the distance $x_{0}{ }^{\prime}-x_{0}$ traveled by the wave front, we write from eq 147 and 148

$$
\frac{d h}{d x_{0}^{\prime}}=\frac{-f_{1} h}{\omega_{0}}\left[1-\frac{3}{4} \frac{\sqrt{g H_{0}}}{\omega_{0}} \frac{h}{H_{0}}\right]
$$

since $d x_{0}{ }^{\prime} / d t=\omega_{1}$; and $3 h \sqrt{g H_{0}} / 4 \omega_{0} H_{0}$ is required to be small with respect to unity. The solution of this differential equation for the initial condition $h=h_{0}$, at $x_{0}^{\prime}=x_{0}$ is

$$
\frac{h}{h_{0}}=\frac{1-\frac{3}{4} \frac{\sqrt{g H_{0}}}{\omega_{0}} \frac{h}{H_{0}}}{1-\frac{3}{4} \frac{\sqrt{g H_{0}}}{\omega_{0}} \frac{h_{0}}{H_{0}}} \exp \left[\frac{f_{1}\left(x_{0}-x_{0}{ }^{\prime}\right)}{\omega_{0}}\right] .
$$

\section{SOLUTION OF THE WAVE EQUATION WHEN SLOPE AND FRICTION ARE NOT NEGLIGIBLE}

The significance of introducing $\omega$, the celerity of a wave-volume element, lies in the fact that the integration of the wave equation, eq 111 becomes equivalent to solving

$$
\frac{\partial h}{\partial t}+\frac{\partial}{\partial x}(h \omega)=0
$$




\section{Journal of Research of the National Bureau of Standards}

where $\omega$ is given by eq 116, or in this particular case by eq 128 . Although the general solution is complicated, if it be assumed that $\partial^{2} h / \partial x^{2}$ is negligible, compared to $6 h / H_{0}{ }^{2} k_{2}$, the problem is considerably simplified. This assumption requires that the curvature of the wave profile be small. We thus obtain from eq 129 and eq 62:

and eq 113 becomes

$$
\omega h=h U_{0}+\left(\omega_{0}-U_{0}\right)\left(1+\frac{3 h}{4 H_{0}}\right) h \mp \sigma f_{1},
$$

$$
\frac{\partial h}{\partial t}+\left[U_{0}+\left(\omega_{0}-U_{0}\right)\left(1+\frac{3 h}{2 H_{0}}\right)\right] \frac{\partial h}{\partial x}=-h f_{1},
$$

since $\partial \sigma / \partial x=\mp h$. The general solution of this differential equation is

$$
x=\left(\omega_{0}+\left(\omega_{0}-U_{0}\right) \frac{3 h}{2 H_{0}} \frac{e^{t f_{1}}-1}{t f_{1}}\right) t+\Phi\left(h e^{t f}{ }_{1}\right),
$$

where $\Phi$ is an arbitrary function. This integral is due to Boussinesq [3, p. 457, eq 392] and Favre speaks of it as one of the Boussinesq integrals [6, p. 106].

In determining the arbitrary function $\Phi$, two cases are of particular interest; (1) the channel is infinite in both directions, and (2) the channel has an entrance and is infinite in only one direction. In the first case, if for $t=0, h$ is a known function of $x,-\infty<x<\infty$, then $\Phi$ can be obtained directly. Let the function be $h=\Psi(x), t=0$. Putting $t=0$ in eq 154 , we have $x=\Phi(h), t=0$, hence in this case $\Phi$ is simply the inverse of $\Psi$. In the second case, $\Phi$ is determined from the boundary condition $h=\psi(t)$ at the channel entrance $x=0$, but in this case the evaluation is indirect and tedious. At $x=0$, eq 154 gives

$$
\Phi\left(h e_{1}^{f_{1}}\right)=-\left[\omega_{0}+\left(\omega_{0}-U_{0}\right) \frac{3 h}{2 H_{0}} \frac{\exp \left(f_{1} t-1\right)}{f_{1} t}\right] t .
$$

In general, the form of the equation does not permit solving explicitly for either $t$ or $h$, hence it is necessary to evaluate $\Phi$ numerically. By considering values of $t$ in the range $-\infty$ to $+\infty$, and the corresponding values of $h$ at the entrance, using $h=\psi(t)$, the magnitude of the independent variable can be computed. Corresponding values of $\Phi$ are obtained from the right-hand member of eq 155 by inserting the same values of $h$ and $t$ used to evaluate $h e^{t s}{ }_{1}$.

\section{INTUMESCENCES PRODUCED BY OPERATING LOCKS IN A CANAL CARRYING WATER}

We now consider a practical example of case (2) above which can be treated by a simpler method. In many cases the canals leading to and from hydroelectric plants are also used for navigation, and a lock is provided to bridge the difference in head utilized by the plant. Whenever this lock is emptied, a positive wave is produced in the downstream canal, and, whenever it is filled, a negative wave is produced in the upstream canal. The former, of course, travels downstream, whereas the latter travels upstream. 
Because of such design considerations as the possibility of overtopping the embankments, it is desirable to predict by computation the magnitude and deformation of these waves. Ordinarily the discharge from the lock is a continuous function of time, the exact relation being obtained from computation or model experiments. The waves produced by operating the lock are small in height and long enough so that the curvature is negligible, and thus eq 154 is applicable.

As far as this analysis is concerned, the lock constitutes a termination of the canal, and the boundary conditions of case (2) apply. The complications of this case are eliminated by a method due to Favre [6, p. 112], which is based on the fact that the distance traveled by the wave is very large compared to its length. The initial shape of the wave is computed for the initial time $t=0$, in the form $h=\Psi(x)$, assuming that the effect of friction is negligible during the short time interval required for the formation of the wave. This function is then used to determine $\Phi$.

Let the discharge at the lock per unit width of channel be represented by the function

$$
q=f(t), x=0 .
$$

As a result of the above, we may also write

$$
t=F(q), x=0 .
$$

It follows from eq 154 that the form of the wave at the moment it is produced and shortly thereafter, neglecting the effect of friction during the genesis of the wave, is represented by

$$
x=\left[\omega_{0}+\left(\omega_{0}-U_{0}\right) \frac{3 h}{2 H_{0}}\right] t+\Phi(h),
$$

since $\operatorname{Lim}_{t 1_{1} \rightarrow 0} \frac{\exp \left(f_{1} t-1\right)}{t f_{1}}=1$. From eq 52 and 117 the mean velocity in a cross section beneath the wave is

$$
U=U_{0}+\frac{\omega-U_{0}}{H_{0}+h} h
$$

Neglecting the effects of friction and curvature in eq 129, this becomes, using eq 62

$$
U=U_{0}+\left(\omega_{0}-U_{0}\right)\left(1+\frac{3 h}{4 H_{0}}\right) \frac{h}{H_{0}+h} .
$$

The discharge per unit width at $x=0$ is thus

$$
q=H U=U_{0}\left(H_{0}+h\right)+\left(\omega-U_{0}\right)\left(1+\frac{3 h}{4 H_{0}}\right) h=f(t), x=0 .
$$

Comparing this equation with eq 157 we have

$$
\begin{aligned}
t & =F\left(U_{0}\left(H_{0}+h\right)+\left(\omega_{0}-U_{0}\right)\left(1+\frac{3 h}{4 H_{0}}\right) h\right), x=0, \\
524586-43-6 &
\end{aligned}
$$


which expresses $h$ as a function of $t$ for $x=0$, and thus serves to determine the arbitrary function $\Phi$ in eq 158. Placing $x=0$ in that equation, we have

and from eq 161

$$
\Phi(h)=-\left[\omega_{0}+\left(\omega_{0}-U_{0}\right) \frac{3 h}{2 H_{0}}\right] t,
$$

$\Phi(h)=-\left[\omega_{0}+\left(\omega_{0}-U_{0}\right) \frac{3 h}{2 H_{0}}\right] F\left(U_{0}\left(H_{0}+h\right)+\left(\omega_{0}-U_{0}\right)\left(1+\frac{3 h}{4 H_{0}}\right) h\right)$.

Since the function $\Phi$ in eq 158 is the same as the function $\Phi$ in eq 154 , we write for the second term in the right-hand member of this equation,

$$
\begin{aligned}
\Phi\left(h e^{f_{1} t}\right)=-\left[\omega_{0}+\left(\omega_{0}-U_{0}\right) \frac{3 h e^{f_{1} t}}{2 H_{0}}\right] F & U_{0}\left(H_{0}+h e^{f_{1} t}\right) \\
& \left.+\left(\omega_{0}-U_{0}\right)\left(1+\frac{3 h e^{f_{1} t}}{4 H_{0}}\right) h e^{f_{1} t}\right) .
\end{aligned}
$$

The solution of a typical problem will now be described in detail. It is desired to find the wave profile in the canal at any instant $t$ when the discharge is given as a function of time, $Q / b=q=f(t), b$ being the width of the free surface of the canal. We shall assume that the discharge first increases and then decreases, as shown in figure 13. This will create a positive surge in the discharge canal and a negative surge in the supply canal. The origin in figure 13 is conveniently chosen to correspond to the instant of maximum discharge. The abscissa $t$ is, of course, expressed in seconds, and the ordinate, $q$, in any convenient unit of discharge per unit width, such as square feet per second. In eq 154 and 164, upon which the solution depends, $U_{0}$ and $\omega_{0}$ will have to be expressed in corresponding units of velocity, such as feet per second.

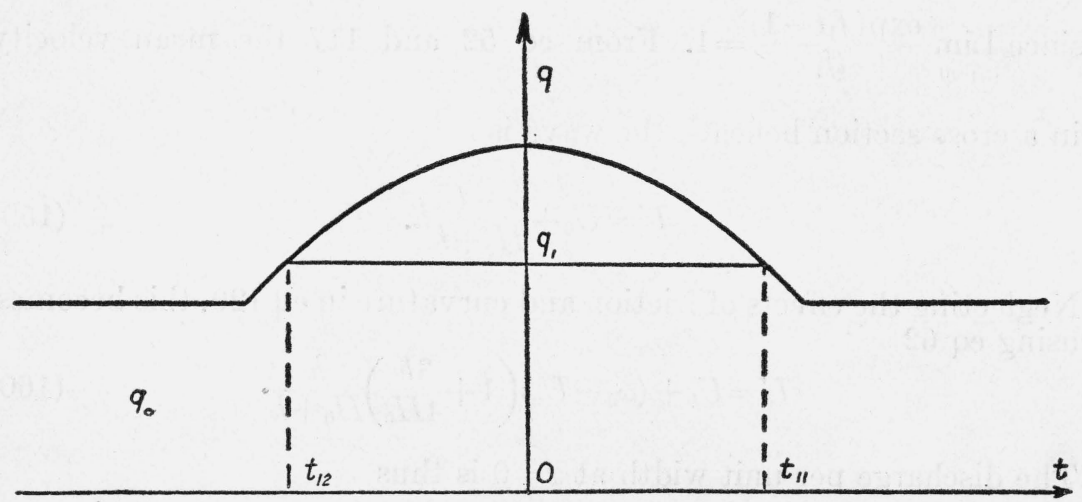

FIG. 13.-Discharge from supply canal as a function of time: $Q / b=q=f(t)$, eq 156, or $t=F(q)$, eq $15 \%$.

The positive surge in the discharge canal will now be considered. The quantities $U_{0}, H_{0}, R_{0}$ and $i$ are determined from measurements of the steady flow. The coefficient of friction, $\lambda_{0}$, is then determined from eq 48. All the quantities needed to determine $\omega_{0}$ from eq 62 , and $f_{1}$ from eq 126 are thus available. Note that the positive sign in eq 62 
is selected, since the wave will be moving downstream. A small positive value of $h$ is now selected, which we shall denote as $h_{1}$. The argument of the function $F$ in eq 164 is computed, using the value of $t$ corresponding to the instant for which the wave profile is desired. As is seen from eq 157, this gives a corresponding value of $q$, which we shall call $q_{1}$. If $q_{1}$ is greater than the maximum value of $q$ which is actually obtained, this signifies that $h_{1}$ was higher than any point of the intumescence. It will be advisable to select $h_{1}$ very small at first and then increase it until the top of the curve in figure 13 is attained. The values of $t$ corresponding to $h_{1}$ will be denoted by $t_{11}$ and $t_{12}$ since, there will generally be two of these, the first positive and the second negative. This is clearly indicated in figure 13. These two values, $t_{11}$ and $t_{12}$, are thus the desired values of the function $F$ occurring in eq 164, and the two values of the double-valued function $\Phi$ can now be obtained by replacing $h$ in the coefficient of $F$ by the quantity $h_{1}$. Turning now to eq 154, replacing $h$ by $h_{1}$ and $t$ by the value corresponding to the instant for which the wave profile is desired, we obtain two values for $x$, corresponding to the original value $h_{1}$, which we shall denote by $x_{11}$ and $x_{12}$. These are the abscissas of the points on the profile having the height $h_{1}$ (see fig. 14). The whole process is repeated for greater heights, $h_{2}, h_{3}, \ldots$, until sufficient points are determined to establish the contour of the wave.

The process for determining the negative surge in the supply channel is exactly the same, except that in eq 62 the minus sign is chosen, since the wave is traveling upstream. The quantities $h_{1}, \ldots$ and $x_{11}, x_{12}, \ldots$, will be negative, as shown in figure 15 .

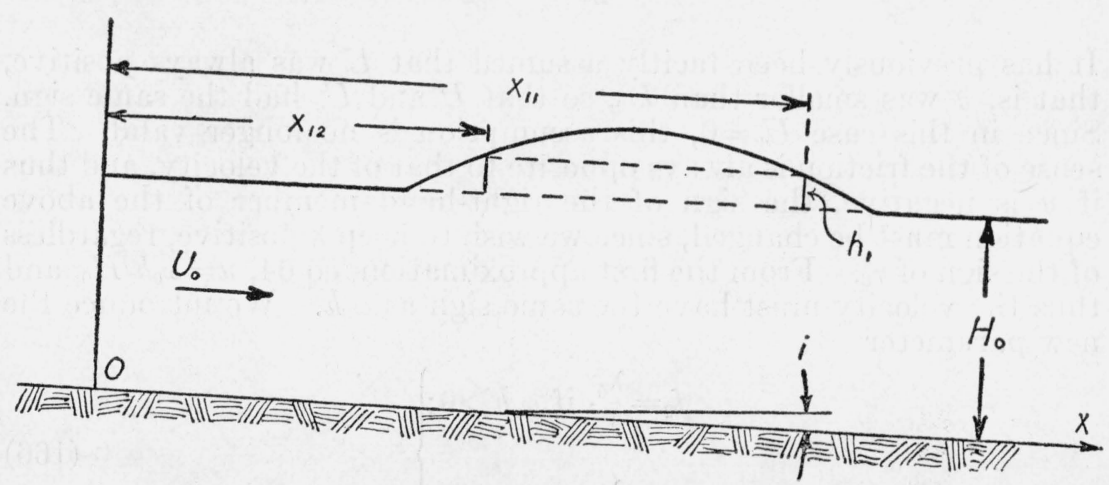

FIGURE 14.-Profile of descending wave in discharge canal.

\section{EFFECT OF FRICTION ON WAVES WHEN THE SLOPE OF THE BED VANISHES}

We now consider case (II) of section IV $-5, i=0$ and $U_{0}=0$. The determination of the positive sense of the $x$-axis on the basis of the conventions in section III-1 fails, since there is no flow. Furthermore, the terms "ascending" and "descending" waves explained in section III-3 fail to have any significance, since we have no method of distinguishing the upstream from the downstream direction. We thus are forced to make an arbitrary choice of the positive $x$-direction, and consider downstream to be in this direction, A descending wave thus 


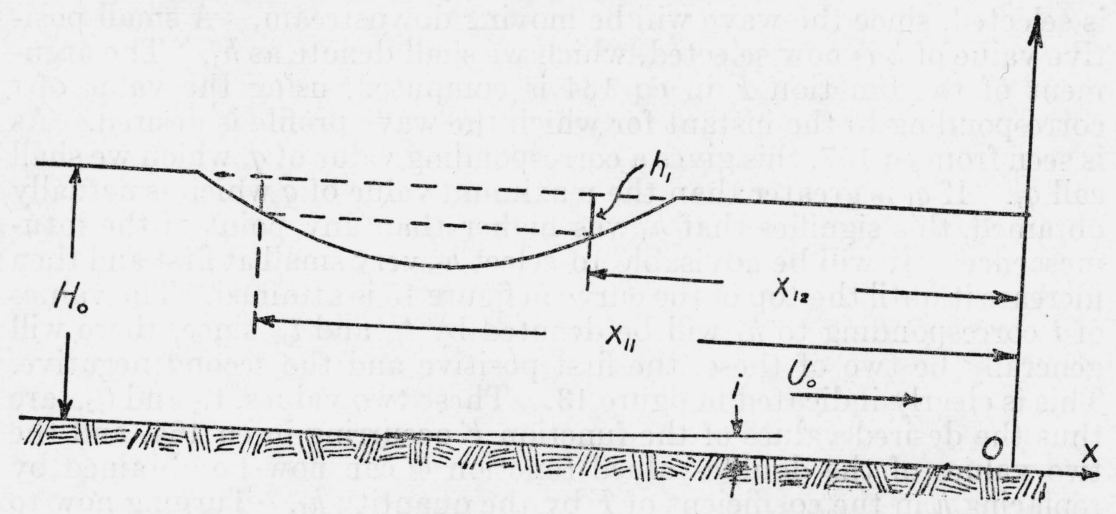

Figure 15.-Profile of ascending wave in supply canal.

becomes one for which $\omega_{0}>0$, and an ascending wave one for which $\omega_{0}<0$, that is to say, a descending wave is propagated in the positive $x$-direction, and an ascending wave is propagated in the opposite direction, since our hypothetical observer is stationary and the downstream direction coincides with the arbitrarily chosen positive sense of the $x$-axis.

Since $i=0$ and $U_{0}=0$, it follows immediately from eq 52 that

$$
g H i-\frac{\lambda}{2} U^{2}=-\frac{\lambda}{2} u^{2} .
$$

It has previously been tacitly assumed that $U$ was always positive, that is, $u$ was smaller than $U_{0}$, so that $U$ and $U_{0}$ had the same sign. Since in this case $U_{0}=0$, this assumption is no longer valid. The sense of the friction is always opposite to that of the velocity, and thus if $u$ is negative, the sign of the right-hand member of the above equation must be changed, since we wish to keep $\lambda$ positive, regardless of the sign of $\tau_{0}$. From the first approximation, eq $64, u=\omega_{0} h / H_{0}$, and thus the velocity must have the same sign as $\omega_{0} h$. We introduce the new parameter

$$
\left.\begin{array}{r}
f_{2}=\frac{\lambda_{0}}{4}, \text { if } \omega_{0} h>0 \\
f_{2}=-\frac{\lambda_{0}}{4}, \text { if } \omega_{0} h<0
\end{array}\right\}
$$

and obtain from eq 165

$$
g H i-\frac{\lambda}{2} U^{2}=-2 \omega_{0}^{2}\left(\frac{h}{H_{0}}\right)^{3} f_{2} .
$$

The difference between $\lambda_{0} u^{2}$ and $\lambda u^{2}$ is a quantity of the order being neglected in this analysis. If we introduce the quantity

$$
\sigma^{\prime}= \pm \int_{x}^{ \pm \infty} h^{2} d x
$$


then since $d \sigma^{\prime} / d x=\mp h^{2}$, we can write eq 167 in the form

$$
g H i-\frac{\lambda}{2} U^{2}= \pm 2 \omega_{0}^{2} f_{2} \frac{d}{d x}\left(\frac{\sigma^{\prime}}{H_{0}^{2}}\right) \text {. }
$$

Substituting this in eq 116 , we have for the velocity of propagation of a volume element

$$
\omega=\omega_{0}\left(1+\frac{2+k_{1}}{4} \frac{h}{H_{0}}+\frac{k_{2} H_{0}^{2}}{6 h} \frac{\partial^{2} h}{\partial x^{2}} \mp \frac{\omega_{0}}{\omega_{0}-U_{0}} \frac{\sigma^{\prime} f_{2}}{h H_{0}^{2}}\right) .
$$

Since in this case $U_{0}=0$, we see from eq 106 that $k_{1}=k_{2}=1$, and thus

$$
\omega=\omega_{0}\left(1+\frac{3 h}{4 H_{0}}+\frac{H_{0}^{2}}{6 h} \frac{\partial^{2} h}{\partial x^{2}} \mp \frac{\sigma^{\prime} f_{2}}{h H_{0}^{2}}\right) .
$$

Consider the sign of $\omega_{0} \sigma^{\prime} f_{2} / h H_{0}{ }^{2}$, which equals $\omega_{0}{ }^{2} \sigma^{\prime} f_{2} / \omega_{0} h H_{0}{ }^{2}$. Since from eq $168, \sigma^{\prime}$ is always positive, it follows that the sign of the above is the same as that of $f_{2} / \omega_{0} h$. But from eq $166 f_{2}$ has the same sign as $\omega_{0} h$, hence the original term is always positive. In accordance with the convention of choice of signs for descending and ascending waves, the choice in eq 171 is always opposite to that of the sign of $\omega_{0}$. Therefore, the effect of friction on the celerity $\omega$ when $i=0, U_{0}=0$, is to diminish its absolute value by an amount which increases with the distance from the head of the wave.

\section{SOLUTION OF THE WAVE EQUATION WHEN THE SLOPE VANISHES BUT FRICTION IS CONSIDERED}

As in section IV-3, the problem is to solve

$$
\frac{\partial h}{\partial t}+\frac{\partial}{\partial x}(h \omega)=0
$$

where $\omega$ is given by eq 116 or, in this particular case, by eq 171. For the reasons mentioned in the same section, we assume that $\partial^{2} h / \partial x^{2}$ is negligible. Equations 171 and 113 thus become

$$
\omega=\omega_{0}\left(1+\frac{3 h}{4 H_{0}} \mp \frac{\sigma^{\prime} f_{2}}{h H_{0}^{2}}\right)
$$

and

$$
\frac{\partial h}{\partial t}+\omega_{0}\left(1+\frac{3 h}{2 H_{0}}\right) \frac{\partial h}{\partial x}=-f_{2} \omega_{0}\left(\frac{h}{H_{0}}\right)^{2},
$$

respectively, since $d \sigma^{\prime} / d x=\mp h^{2}$. Assuming that the wave is everywhere positive or everywhere negative, then $f_{2}$ is constant, and the general integral of the above differential equation is

$$
x=\omega_{0}\left[1+\frac{3 h}{2 H_{0}} \frac{\log \left(1-\frac{f_{2} \omega_{0}}{H_{0}^{2}} h t\right)}{-\frac{f_{2} \omega_{0}}{H_{0}{ }^{2}} h t}\right] t+\Phi\left[\frac{h}{1-\frac{f_{2} \omega_{0}}{H_{0}{ }^{2}} h t}\right],
$$

where $\Phi$ is an arbitrary function. This is Boussinesq's second integral [3, p. 454 eq 393].

Regarding the determination of this arbitrary function, we consider the same two cases as in section IV-8: (1) the channel is infinite in 
both directions, and (2) the channel has an entrance which is infinite in only one direction. In the first case, if for $t=0, h$ is a known function of $x,-\infty<x<\infty$, then $\Phi$ can be obtained directly. Let the function be $h=\Psi(x), t=0$. Putting $t=0$ in eq 174 we have $x=\Phi(h), t=0$, hence in this case $\Phi$ is simply the inverse of $\Psi$. In the second case, $\Phi$ is determined from the boundary condition $h=\psi(t)$ at the channel entrance $x=0$; but in this case the evaluation is indirect and tedious. At $x=0$, eq 174 gives

$$
\Phi\left(\frac{h}{1-\frac{f_{2} \omega_{0}}{H_{0}^{2}} h t}\right)=-\omega_{0}\left[1+\frac{3 h}{2 H_{0}} \frac{\log \left(1-\frac{f_{2} \omega_{0}}{H_{0}^{2}} h t\right)}{\frac{-f_{2} \omega_{0}}{H_{0}^{2}} h t}\right] t .
$$

In general, the form of the equation does not permit solving explicitly for either $t$ or $h$, hence it is necessary to evaluate $\Phi$ numerically. By considering values of $t$ in the range $-\infty$ to $+\infty$, and the corresponding value of $h$ at the entrance, using $h=\psi(t)$, the magnitude of the independent variable can be computed. Corresponding values of $\Phi$ are obtained from the right-hand member of eq 175 by inserting the same values of $h$ and $t$ used to evaluate

$$
\frac{h}{1-\frac{f_{2} \omega_{0}}{\bar{H}_{0}{ }^{2}} h t} .
$$

These methods are precisely analogous to those discussed in connection with eq 155 .

\section{INTUMESCENCE PRODUCED BY OPERATING LOCKS IN AN ORDINARY CANAL}

In contrast to canalized rivers and hydroelectric canals, such as discussed in section IV-9, ordinary canals contain still water except when disturbances are set up by the operation of the locks. The general considerations at the beginning of section IV-9 (through eq 157) also apply to this case, except that $i=0$ and $U_{0}=0$, and hence we must consider case (2) of eq 174.

The discharge per unit width at the lock is again represented by

$$
q=f(t), \quad x=0,
$$

or by the inverse function

$$
t=F(q), \quad x=0 .
$$

Neglecting the effect of friction during the formation of the wave and shortly thereafter, we obtain from eq 174

since

$$
x=\omega_{0}\left(1+\frac{3 h}{2 H_{0}}\right) t+\Phi(h),
$$


For the mean velocity in a cross section beneath the wave, eq 52 , 117,171 give

$$
U=\omega_{0}\left(1+\frac{3 h}{4 H_{0}}\right) \frac{h}{H_{0}+h},
$$

since $U_{0}=0$, and friction and curvature are neglected. The discharge per unit width at $x=0$ is, therefore,

$$
q=H U=\omega_{0} h\left(1+\frac{3 h}{4 H_{0}}\right), x=0 .
$$

Substituting this value of $q$ in eq 157 , we obtain

$$
t=F\left(\omega_{0} h\left(1+\frac{3 h}{4 H_{0}}\right)\right), x=0
$$

But from eq 176

$$
\Phi(h)=-\omega_{0}\left(1+\frac{3 h}{2 H_{0}}\right) t, x=0,
$$

or from eq 179

$$
\Phi(h)=-\omega_{0}\left(1+\frac{3 h}{2 H_{0}}\right) F\left(\omega_{0} h\left(1+\frac{3 h}{4 H_{0}}\right)\right) .
$$

Since this function $\Phi$ in eq 176 is the same as the function $\Phi$ in eq 174 , we have for the right-hand member of this equation,

$$
\begin{aligned}
\Phi\left(\frac{h}{1-\frac{f_{2} \omega_{0}}{H_{0}{ }^{2}} h t}\right)=-\omega_{0}\left[1+\frac{3 h}{2 H_{0}\left(1-\frac{f_{2} \omega_{0}}{H_{0}} h t\right)}\right] \\
F\left(\frac{\omega_{0} h}{1-\frac{f_{2} \omega_{0}}{H_{0}^{2}} h t}\left[1+\frac{3 h}{4 H_{0}\left(1-\frac{f_{2} \omega_{0}}{H_{0}^{2}} h t\right)}\right]\right) .
\end{aligned}
$$

Equations 174 and 182 are used to determine the wave profile at any instant $t$, as is described in section IV-9.

\section{LIMITATIONS OF BOUSSINESQ SOLUTIONS}

It is necessary to emphasize the fact that the second-order theory, which is based on the concept of the velocity of propagation, $\omega$, of a volume element, is subject to certain important restrictions. The most general expression for $\omega$ is given in eq 116. In deriving this equation, the important assumption was made that $\omega / \omega_{0}$ is nearly equal to unity.

We shall now turn our attention to the specialized equations derived from eq 116. These are eq 118, 128, 171. Examination shows that in each case, $\omega / \omega_{0}=1+$ (correction terms). The restriction on the theory thus requires that these terms shall be small compared to unity. The first term, which is common to all equations, is $\left(2+k_{1}\right) h$ $/ 4 H_{0}$. This merely requires that $h \ll 4 H_{0} /\left(2+k_{1}\right)$. The second term, which expresses the effect of curvature, requires that $\left(\partial^{2} h / \partial x^{2}\right) / h$ $\ll 6 / H_{0}{ }^{2} k_{2}$. We see from this, that $\left(\partial^{2} h / \partial x^{2}\right)$ must vanish to a higher order than $h$.

The third term, which appears only in eq 128 and 171, expresses the effect of friction. These two equations are applicable only when 


\section{2 Journal of Research of the National Bureau of Standards}

$\sigma f_{1} / \omega_{0} h$ or $\sigma^{\prime} f_{2} / h H_{0}^{2}$, respectively, is small. But $\sigma$ and $\sigma^{\prime}$ can be made as large as we please merely by considering longer and longer intumescences, and if the intumescence has a finite volume, $h$ approaches zero at the tail whereas $\sigma$ and $\sigma^{\prime}$ remain finite. This shows that the term under consideration may be large, or even infinite. In view of this fact, it may be expected that these solutions will give correct values only in the frontal portion of the wave where $\sigma / h$ and $\sigma^{\prime} / h$ are small. It should be noted that this remark applies with equal force to the Boussinesq integrals, eq 154 and 174 .

\section{REFERENCES}

[1] G. H. Keulegan and G. W. Patterson, Mathematical theory of irrotational translation waves, J. Research NBS 24, 47 (1940) RP1272.

[2] H. Bazin, Recherches experimentales sur la propagation des ondes, Mém. divers Savants a L'Acad. des Sci. 19, 495 (1865).

[3] J. Boussinesq, Essai sur la théorie des eaux courantes. Mém. divers Savants a L'Acad. des Sci. 23, 1, (1877).

[4] R. E. Horton, Channel Waves Subject Chiefly to Momentum Control, U. S. Department of Agriculture, Soil Conservation Service Technical Publication 16, (mimeographed, 1938).

[5] G. H. Keulegan, Laws of turbulent flow in open channels. J. Research NBS 21, 707 (1938) RP1151.

[6] H. Favre, Étude Théorique et Expérimentale des Ondes de Translation dans les Canaux Découverts (Dunod, Paris, 1935).

[7] O. Reynolds, On the dynamical theory of incompressible viscous fluids and the determination of the criterion. Phil. Trans. [A] 186, 123 (1895).

[8] H. Lamb, Hydrodynamies, [ed. 5] (Cambridge University Press, Cambridge, England, 1924).

[9] H. L. Dryden, F. D. Murnaghan, H. Bateman, Report of the committee on hydrodynamics, Bul. National Research Council, No. 84 (1922).

[10] S. Goldstein, ed., Modern Developments in Fluid Dynamics, 1, (Oxford University Press, Oxford, England, 1938).

[11] H. Rouse, Fluid Mechanies for Hydraulic Engineers (McGraw-Hill Book Co., Inc., New York, N. Y., 1938).

[12] G. H. Keulegan, Equation of motion for the steady mean flow of water in open channels, J. Research NBS \%9, 97 (1942) RP1488.

[13] J. Hadamard, Leçons sur la Propagation des Ondes et les Équations de l'Hydrodynamique (Librarie Scientifique A. Hermann, Paris, 1903).

[14] J. Nikuradse, Strömungsgesetze in rauhen Rohren (Verein Deutscher Ingenieure, Forschungsheft 361, 1933).

[15] H. Bazin, Experiences sur la propagation des ondes le long d'un cours d'eaux torrenteux . . . ., Compt. rend. 100, 1492 (1885).

[16] G. H. Keulegan and G. W. Patterson, A criterion for instability of flow in steep channels, Trans. Am. Geophys. Union, part II, 594, (1940).

Washington, March 1, 1943. 
NATIONAL BUREAU OF STANDARDS,

WASHINGTON, D. C.

Send me the Mathematical Tables marked $\mathrm{X}$ below. I enclose remittance ${ }^{1}$ to cover the cost.

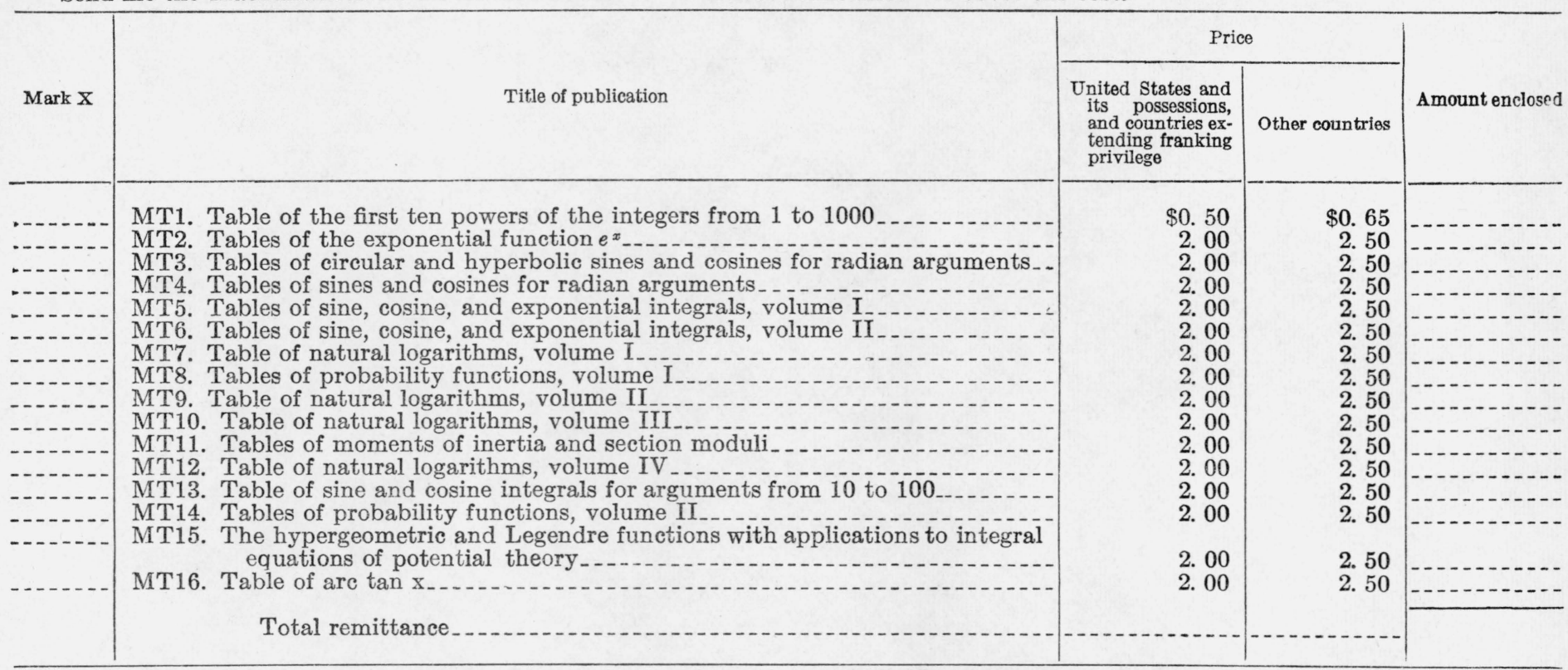

${ }^{1}$ Remittance should be in form of post-office money order, or check, and made payable to the order of the "National Bureau of Standards" in United States currency.

Send to

Number and Street.

City and State. 


\section{MATHEMATICAL TABLES}

Attention is invited to a series of publications prepared by the Project for the Computation of Mathematical Tables conducted by the Federal Works Agency, Work Projects Administration for the City of New York under the sponsorship of the National Bureau of Standards. The tables which have been made avail. able through the National Bureau of Standards are listed below.

There is included in this list a publication on the hypergeometric and Legendre functions (MT15), prepared by the Bureau.

MT1. Table or the First Ten Powers or thr Intrgers From 1 to 1000:

(1938) VIII + 80 pages; heavy paper cover. 50 cents.

MT2. Tables of the Exponential Function $e^{x}$.

The ranges and intervals of the argument and the number of decimal places in the entries are given below:

$\begin{array}{ccc}\text { Range of } x & \text { Interval of } x & \text { Decimal given } \\ -2.5000 \text { to } 1.0000 & 0.0001 & 18 \\ 1.0000 \text { to } 2.5000 & .0001 & 15 \\ 2.500 \text { to } 5.000 & .001 & 15 \\ 5.00 \text { to } 10.00 & .01 & 12\end{array}$

(1939) XV+535 pages; bound in buckram, $\$ 2.00$.

MT3. Tables of Circular and Hyprrbolic Sings and Cosings for Radian Arguments:

Contains 9 decimal place values of $\sin x, \cos x, \sinh x$ and $\cosh x$ for $x$ (in radians) ranging from 0 to 2 at intervals of 0.0001 .

(1939) XVII + 405 pages; bound in buckram, $\$ 2.00$.

MT4. Tables or Stnes and Cosines for Radian Arguments:

Contains 8 decimal place values of sines and cosines for radian arguments ranging from 0 to 25 at intervals of 0.001 .

(1940) XXIX + 275 pages; bound in buckram, $\$ 2.00$.

MT5. Tables of Sing, Cosine, and Exponential Intrgrals, Volume I:

Values of these functions to 9 places of decimals from 0 to 2 at intervals of 0.0001 .

(1940) XXVI + 444 pages; bound in buckram, $\$ 2.00$.

MT6. Tables of Sine, Cosine, and Exponential Intggrals, Volume II:

Values of these functions to 9,10 , or 11 significant figures from 0 to 10 at intervals of 0.001 with auxiliary tables.

(1940) XXXVII+225 pages; bound in buckram, $\$ 2.00$.

MT7. Tablr op Natural Logarithm, Volume I:

Logarithms of the integers from 1 to 50,000 to 16 places of decimals.

(1941) XVIII + 501 pages; bound in buckram, $\$ 2.00$.

MT8. Tables of Probability Functions, Volume I:

Values of these functions to 15 places of decimals from 0 to 1 at intervals of 0.0001 and from 1 to 5.6 at intervals of 0.001 .

(1941) XXVIII + 302 pages; bound in buckram, $\$ 2.00$.

[Continued on p. 4 of cover] 
MT9. Table of Natural Logarithms, Volume II:

Logarithms of the integers from 50,000 to 100,000 to 16 places of decimals.

(1941) XVIII + 501 pages; bound in buckram, $\$ 2.00$.

MT10. Table of Natural Logarithms, Volumb III:

Logarithms of the decimal numbers from 0.0001 to 5.0000 , to 16 places of decimals.

(1941) XVIII + 501 pages; bound in buckram, $\$ 2.00$.

MT11. Tables of the Momrnts of Inertia and Section Moduli of Ordinary Anglzs, Chan. nels, and Bulb Angles with Certain Plate Combinations:

(1941) XIII + 197 pages; bound in green cloth. $\$ 2.00$.

MT12. Table of Natural Logarithms, Volume IV:

Logarithms of the decimal numbers from 5.0000 to 10.0000 , to 16 places of decimals.

(1941) XXII + 506 pages; bound in buckram, $\$ 2.00$.

MT13. Tabir or Sine and Cosine Intrgrals for Arguments From 10 to 100:

(1942) XXXII + 185 pages, bound in buckram, $\$ 2.00$.

MT14. Tables of Probability Functions, Volume II:

Values of these functions to 15 places of decimals from 0 to 1 at intervals of 0.0001 and from 1 to 7.8 at intervals of 0.001 .

(1942) XXI + 344 pages; bound in buckram, $\$ 2.00$.

MT15. The hypergeometric and Legendre functions with applications to integral equations of potential theory. By Chester Snow, National Bureau of Standards. Reproduced from original handwritten manuscript.

(1942) VII+319 pages; bound in heavy paper cover. $\$ 2.00$.

MT16. TABLr op Arc Tan X:

Table of inverse tangents for positive values of the angle in radians. Second central differences are included for all entries.

$\begin{array}{rcc}\text { Range of } x & \begin{array}{c}\text { Interval between } \\ \text { successive arguments }\end{array} \\ 0 \text { to } & 7 & 0.001 \\ 7 \text { to } & 50 & .01 \\ 50 \text { to } 300 & .1 \\ 300 \text { to } 2,000 & 1 \\ 2,000 \text { to } 10,000 & 10\end{array}$

(1942) XXV+169 pages; bound in buckram, $\$ 2.00$.

MT17. Miscellaneous Physical Tables:

Planck's radiation functions (Originally published in the Journal of the Optical Society of America, February 1940); and

Electronic functions.

(1941) VI + 58 pages, bound in buckram, $\$ 1.50$.

MT18. Table of the Zeros of the Legendre Polynomials of Order 1-16 and the Weight Coefficients for Gauss's Mechanical Quadrature Formula.

(Reprinted from Bull. Amer. Mathemical Society, October 1942.)

5 pages. 25 cents.

Payment is required in advance. Make remittance payable to the "National Bureau of Standards," and send with order, using the blank form facing page 3 of the cover.

The prices are for delivery in the United States and its possessions and in countries extending the franking privilege. To other countries the price of MT1 is 65 cents; that of MT2 to MT16, inclusive, is $\$ 2.50$ each; MT17, $\$ 1.75$; MT18, 30 cents; remittance to be made payable in United States currency.

Copies of these publications have been sent to various Government depositories throughout the country, such as public libraries in large cities, and colleges and universities, where they may be consulted.

A mailing list is maintained for those who desire to receive announcements regarding new tables as they become available. 